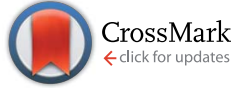

Cite this: RSC Adv., 2017, 7, 9780

Received 30th December 2016 Accepted 30th January 2017

DOI: $10.1039 / \mathrm{c} 6 \mathrm{ra} 28855 \mathrm{c}$

rsc.li/rsc-advances

\title{
Phosphinito palladium(II) complexes as catalysts for the synthesis of 1,3-enynes, aromatic alkynes and ynones
}

\author{
R. E. Islas, ${ }^{a}$ J. Cárdenas, ${ }^{* a}$ R. Gaviño, ${ }^{a}$ E. García-Ríos, ${ }^{a}$ L. Lomas-Romero ${ }^{b}$ \\ and J. A. Morales-Serna*b
}

\begin{abstract}
An air-stable phosphinito palladium(II) complex (Ph1-Phoxide) has been found to be an efficient catalyst in the formation of $\mathrm{C}-\mathrm{C}$ bonds. The coupling of terminal alkynes formed gem-1,3-enynes as the only reaction products. Aromatic alkynes can be synthesized from the coupling of terminal alkynes and haloaromatic compounds (Sonogashira coupling). The phosphinito palladium(॥) complex also catalyses the coupling between acyl chlorides and terminal alkynes (Sonogashira coupling), furnishing ynones in excellent yields.
\end{abstract}

\section{Introduction}

Secondary phosphine oxides (SPOs) ${ }^{\mathbf{1}}$ are a class of preligands $^{2}$ that are valued for their stability in air and in moisture. ${ }^{3}$ Because of the tautomeric equilibrium between the pentavalent phosphorus oxide $\mathbf{1}$ and the trivalent phosphinous acid 2 (Scheme 1), ${ }^{4}$ SPOs form hydrogen bondstabilized bidentate ligands. ${ }^{2,5}$ The shift of this equilibrium in favour of either species, and therefore the formation of transition metal complex $\mathbf{4}$, depends on metal coordination, ${ }^{6}$ the solvents used in the process ${ }^{7}$ and the phosphine substituents. ${ }^{8}$ The formation of $\mathbf{4}$ can be rationalized as the coordination of phosphinous acid 2 and phosphinito ligand 3, which in turn, have their origin in two SPOs. A strong symmetric hydrogen bridge is formed in the presence of a metal, leading to the formation of mononuclear metal complex 4 (Scheme 1).

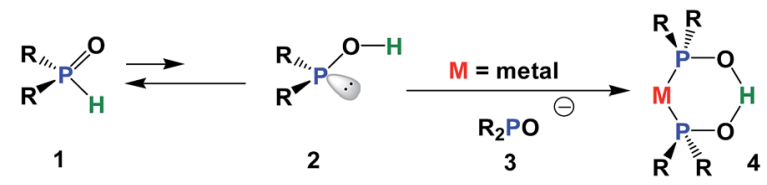

Scheme 1 Tautomerization of SPOs and chelate formation.
Many phosphinito transition metal complexes have been synthesized, ${ }^{2}$ including complexes of palladium, ${ }^{9}$ platinum, ${ }^{\mathbf{1 0}}$ molybdenum, ${ }^{5 e}$ nickel, ${ }^{5 b}$ gold, ${ }^{3 c}$ rhodium $^{5 d, 6 e}$ and ruthenium. ${ }^{11}$ Applications of SPO Pd complexes in catalysis include Negishi cross-coupling (POPd1, POPd5 and POPd7), ${ }^{12}$ conjugate addition of arylsiloxanes ${ }^{\mathbf{1 3}}$ to $\alpha, \beta$-unsaturated carbonyl compounds (POPd1), ${ }^{14}$ Suzuki-Miyaura (POPd1, POP7 and Ph1-Phoxide) ${ }^{15}$ and Stille and Hiyama-type coupling (POPd1) ${ }^{16}$ (Fig. 1).

SPO Pd complex 8 exhibits high catalytic activity in Heck-type reactions as well as high stability towards moisture and air. ${ }^{17}$

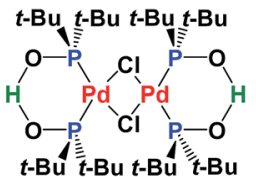

5 (POPd1)

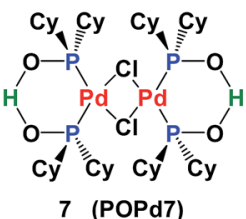

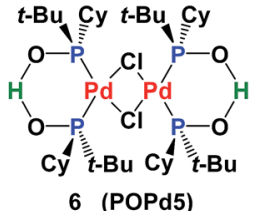

6 (POPd5)

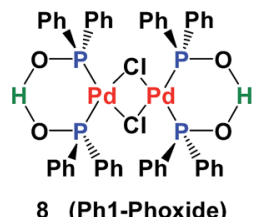

Fig. 1 Structures of SPO Pd complexes.

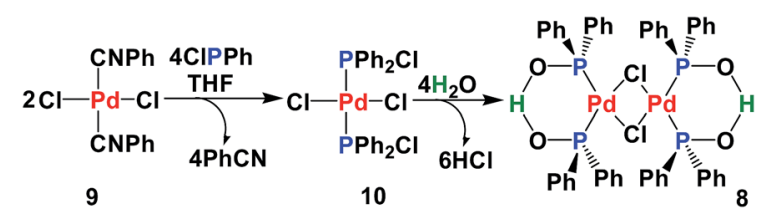

Scheme 2 Synthesis of the SPO Pd complex Ph1-Phoxide. anstituto de Quimica, Universidad Nacional Autónoma de México, Circuito Exterior,
Ciudad Universitaria, Ciudad de México, 04510, Mexico. E-mail: rjcp@unam.mx;
Fax: +52555616 2217; Tel: +525556224413
${ }^{b}$ Departamento de Quimica, Universidad Autónoma Metropolitana-Iztapalapa, Av. San
Rafael Atlixco No. 186, Ciudad de México, C. P. 09340, Mexico. E-mail: joseantonio.
moralesserna@xanum.uam.mx; Fax: +52 $5553189000 ;$ Tel: +52 5553189593 


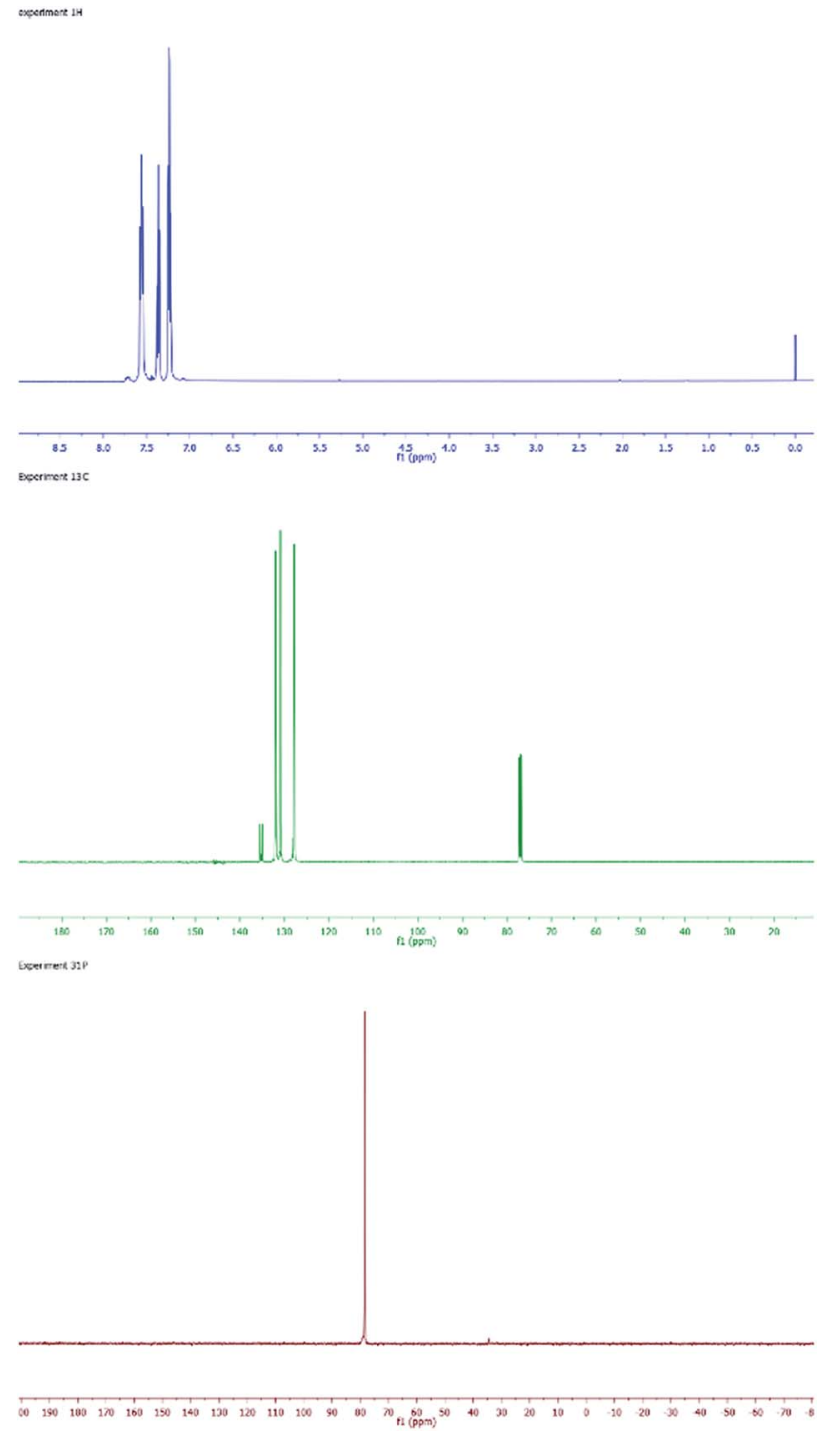

Fig. 2 NMR spectra of complex $8:{ }^{1} \mathrm{H}$ (blue), ${ }^{13} \mathrm{C}$ (green) and ${ }^{31} \mathrm{P}($ red).

The SPOs Pd complex 8 was first synthesized by Dixon ${ }^{\mathbf{1 8}}$ in 1971 and then later synthesized and crystallized by many groups. ${ }^{19}$ From a structural view point, 8 can be considered as a dimer of complex 4. However, our synthesis of $\mathbf{8}$ was achieved by hydrolysis of the palladium chlorodiphenylphosphine complex 10 (Scheme 2). Fig. 2 shows the NMR spectra $\left({ }^{1} \mathrm{H},{ }^{13} \mathrm{C}\right.$ and $\left.{ }^{31} \mathrm{P}\right)$ of complex 8, which is a homogeneous yellow solid.

Driven by our interest in the catalytic properties of SPO Pd complex 8 in organic reactions and its application in the synthesis of natural products, ${ }^{17}$ we report herein the results obtained in the application of complex $\mathbf{8}$ as a catalyst for the synthesis of 1,3-enynes, aromatic-alkynes and ynones (Sonogashira coupling). Additionally, we report the utility of complex 8 in the synthesis of anemarchalconyn, a natural product isolated from the rhizomes of Anemarrhena asphodeloides. To the best of our knowledge, the use of the Pd SPO complex $\mathbf{8}$ in these reactions has not been reported previously.

\section{Results and discussion}

\subsection{Synthesis of 1,3-enynes}

Initially, we were interested in evaluating the ligation properties of the secondary phosphine oxides in complex 8, with the thought that the sterically demanding groups in that complex influence the selectivity of the reaction. The first reaction examined was the coupling of two alkynes to obtain 1,3enynes. ${ }^{20,21}$ As shown in Table 1, the dimerization reaction of alkynes in the presence of $5 \mathrm{~mol} \%$ complex 8 , NaOAc and toluene as the solvent at $100{ }^{\circ} \mathrm{C}$ affords 1,3 -enynes $\mathbf{1 2 a - 1 2 j}$ in good to excellent yield. In all cases, the use of a microwave reactor allowed for a significant reduction in reaction time, from $18 \mathrm{~h}$ to 30 minutes, without affecting yield.

This alkyne dimerization reaction can result in three different isomers, gem, $(Z)$ and $(E)$, depending on the reaction conditions and/or the catalyst. In this case, only the gem isomer was formed, which suggests that the presence of the sterically bulky group on the SPO has an effect on the regioselectivity of the reaction. The same reaction in the presence of $\mathrm{Pd}(\mathrm{OAc})_{2}$ and $\mathrm{PPh}_{3}$ gives a mixture of the three isomers.

The proposed mechanism for the enyne formation is shown in Scheme 3. The first step involves the rupture of the Ph1Phoxide 8 by sodium acetate to form the catalytic precursor $\mathbf{A}$. This precursor promotes ligand exchange with the alkyne substrates $\mathbf{B}$ and $\mathrm{C}-\mathrm{C}$ coupling between them, generating species $\mathbf{C}$. The enyne is obtained by insertion of another alkyne substrate molecule, regenerating the proposed catalytic species B (Scheme 3$).^{20}$

Table 1 Synthesis of 1,3-enynes ${ }^{a, b}$

11

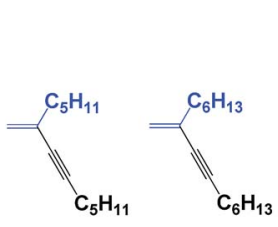

$12 \mathrm{a}(92 \%)$

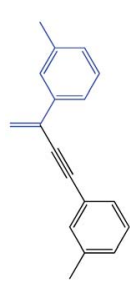

$12 f(83 \%)$

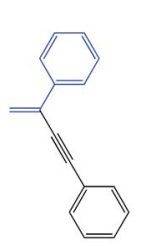

$12 b(91 \%)$

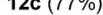

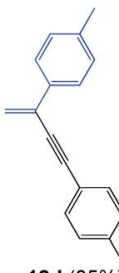

$12 \mathrm{~d}(85 \%)$

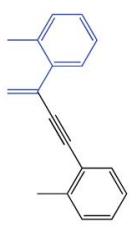

$12 \mathrm{e}(92 \%)$

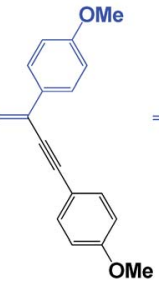

12h $(85 \%)$

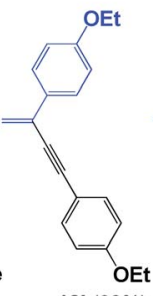

$12 \mathbf{i}(82 \%)$

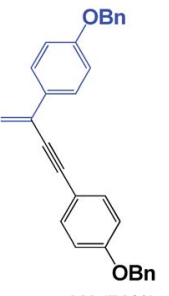

12j (76\%)
${ }^{a}$ Reagents: alkyne 11 (1 mmol), Pd complex 8 (5\% mmol), NaOAc (2 $\mathrm{mmol})$, toluene $(25 \mathrm{~mL})$. ${ }^{b}$ Yield of isolated product after chromatographic purification. 


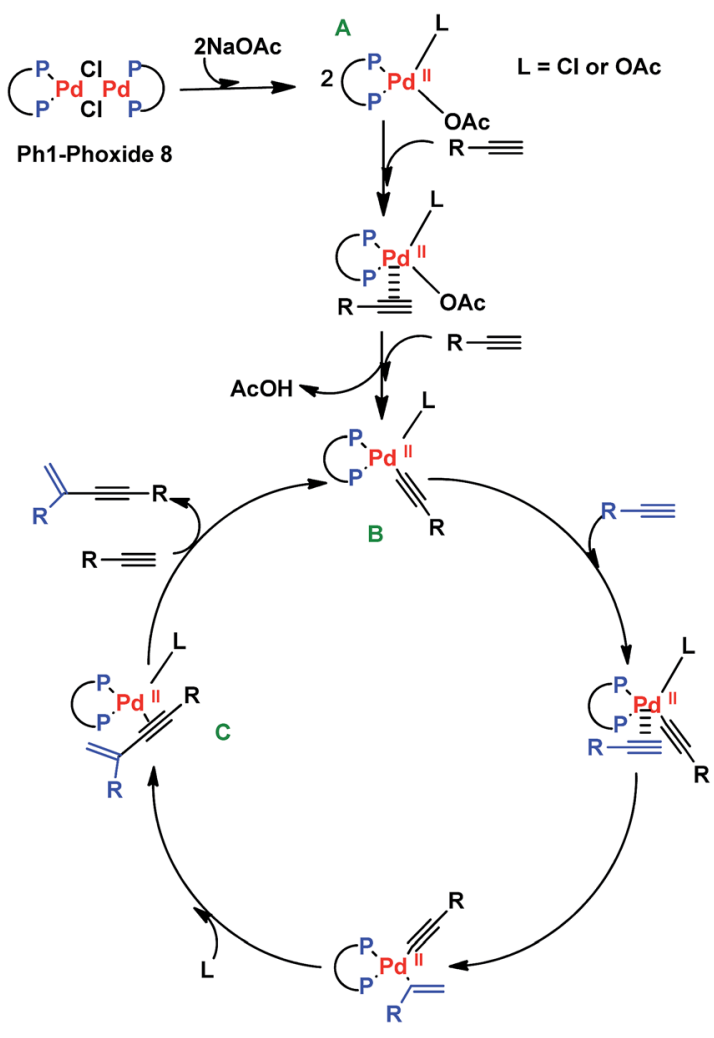

Scheme 3 Proposed mechanism for 1,3-enynes formation.

\subsection{Synthesis of aromatic-alkynes}

Following these results, we extended the use of SPO Pd complex 8 towards the coupling of terminal alkynes to aromatic rings (Sonogashira coupling). ${ }^{22,23}$ Iodobenzene derivatives were tested first, as they are often used as model substrates for a class of reactions. The phenyl-alkynes 14a-14f were isolated in excellent yields ( $90 \%$, Table 2). The yield decreased to $80 \%$ when substituted bromobenzenes were used. No product was observed with chlorobenzene derivatives. When the reactions were carried out in the absence of CuI, only alkyne dimers were isolated. Again, the use of a microwave reactor allowed for a significant reduction in reaction time, from $18 \mathrm{~h}$ to 20 minutes, without affecting yield.

The reaction also works with halogenated heterocycles. For example, 2- and 3-halopyridines were alkynylated under the same reaction conditions; both products were obtained in excellent yield. Notably, alkynylation of the 3-position of pyridine is known to be a difficult reaction, but this reaction proceeds smoothly in the presence of complex 8 ( $90 \%$ for iodoand $80 \%$ for bromo-substituted aryls). The reaction also tolerates halothiophenes, providing the alkyne-aryl adduct in excellent yield (Table 2).

\subsection{Synthesis of ynones}

SPO complex 8 was also applied in the synthesis of $\alpha, \beta$-acetylenic carbonyl compounds (ynones), using highly reactive acyl chlorides as substrates. Ynones are often employed as key intermediates in natural product synthesis. ${ }^{24,25}$ In this case, the
Table 2 Synthesis of phenyl-alkynes ${ }^{a, b}$

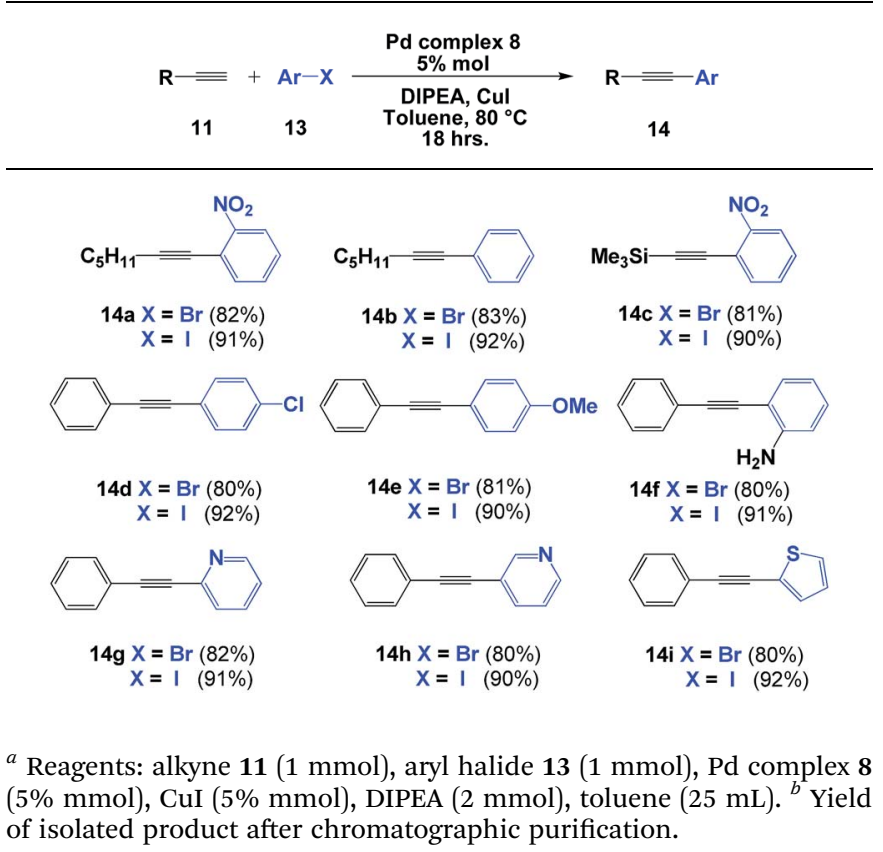

ynones were obtained in excellent yield when the reaction was performed in the presence of triethylamine in toluene at $100^{\circ} \mathrm{C}$; the scope of the reaction was then examined using these optimum reaction conditions. Ynones 16a-16i were obtained in high yield (Table 3). When the reactions were carried out with microwave heating, similar yields were obtained in $1 \mathrm{~h}$.

It is noteworthy that when the reaction was carried out with an alkyl acyl chloride (butyryl chloride 17), the only product observed was the $\beta$-lactone 19. This product could be explained

Table 3 Synthesis of ynones ${ }^{a, b}$
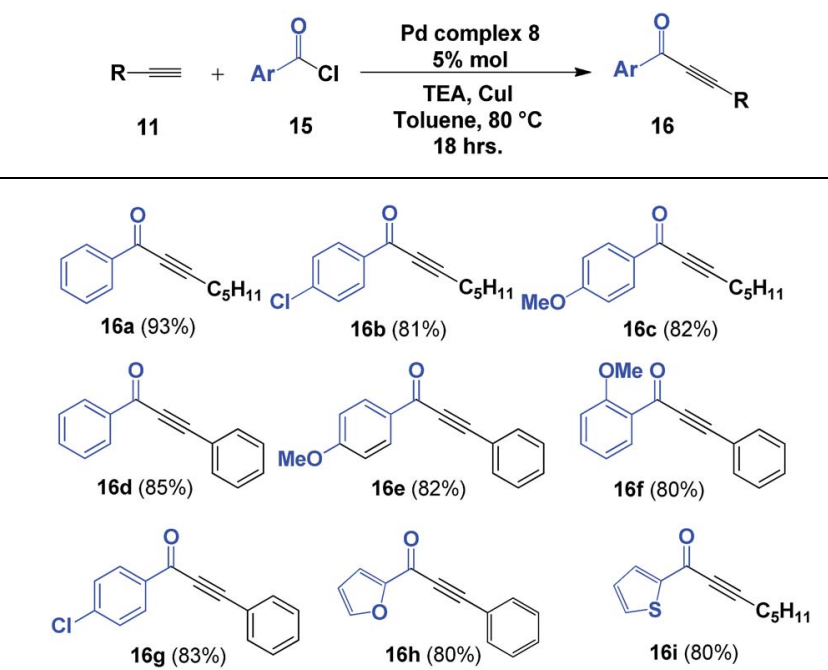

${ }^{a}$ Reagents: alkyne 11 (1 mmol), acyl chloride 15 (1 mmol), Pd complex 8 $(5 \% \mathrm{mmol})$, CuI $(5 \% \mathrm{mmol})$, TEA $(2 \mathrm{mmol})$, toluene $(25 \mathrm{~mL}) .{ }^{b}$ Yield of isolated product after chromatographic purification. 


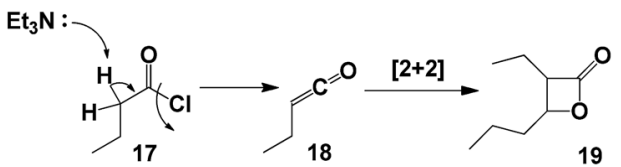

Scheme 4 Reaction of alkyl acyl chloride in presence of TEA.

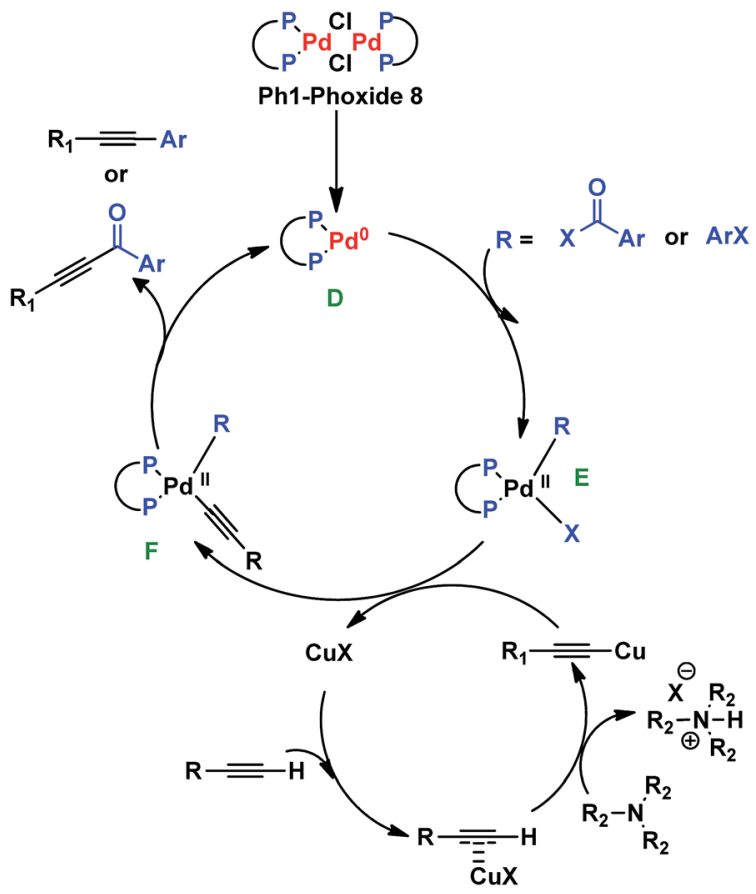

Scheme 5 Proposed mechanism for aromatic-alkynes and ynones formation.

as a result of $[2+2]$ cycloaddition of ketene 18 , which was formed from alkyl acyl chloride 17 in presence of triethtylamine (Scheme 4). ${ }^{26}$

The mechanism involved in both ynones and phenyl-alkynes synthesis is proposed as follows: the formation of the catalytic species $\mathbf{D}$ is performed in situ by rupture of the dimeric complex 8. Then species $\mathbf{E}$ is formed by oxidative addition of an acyl chloride or an aryl halide. A trasmetallation reaction between the complex $\mathbf{E}$ and the copper acetylide generates species $\mathbf{F}$. Finally, the products are formed by reductive elimination, which regenerates the palladium catalyst (Scheme 5 ). ${ }^{22}$

\subsection{Synthesis of anemarchalconyn}

With optimized conditions for ynone formation and aryl alkynylation, we decided to apply our methodology to synthesis of a natural product that would utilize SPO complex 8 in two separate steps. Anemarchalconyn (16k, Scheme 6) was isolated from the rhizomes of Anemarrhena asphodeloides and has inhibitory effects on the adipogenic differentiation of preadipocyte 3T3-L1 cells, with an IC50 value of $5.3 \mu \mathrm{M} .{ }^{27}$ The synthesis began with the methylation of 4-iodophenol 20, followed by the addition of ethynyltrimethylsilane to 4-iodoanisole 21 in the presence of palladium complex 8, generating
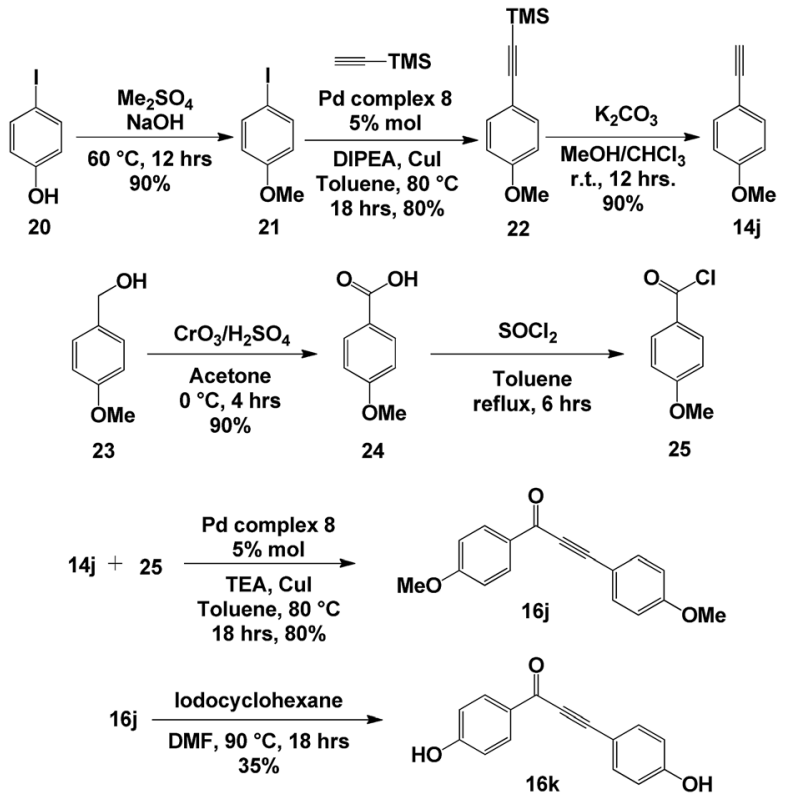

Scheme 6 Synthesis of anemarchalconyn.

intermediate 22 in $80 \%$ yield. The silyl group was removed using basic conditions to give alkyne $14 \mathbf{j}$ in $90 \%$ yield. Oxidation of $p$-anisyl alcohol 23, followed by activation of carbonyl group, afforded acyl chloride 25 . The key step in the synthesis was the coupling between alkyne $\mathbf{1 4} \mathbf{j}$ and acyl chloride 25 catalysed by the palladium complex $\mathbf{8}$, giving ynone $\mathbf{1 6 j}$ in $80 \%$ yield. Finally, removal of the methyl ether protecting group furnished anemarchalconyn (16k) in 35\% yield (Scheme 1). The ${ }^{1} \mathrm{H}$ and ${ }^{13} \mathrm{C}$ NMR spectroscopic data matched those reported for the natural product. $^{27}$

\section{Conclusions}

In conclusion, we have demonstrated that an air-stable palladium(II) phosphinito complex 8 (Ph1-Phoxide), was able to catalyse the regioselective dimerization of alkynes to obtain gem-1,3-enynes, the coupling of haloaromatics and acyl chlorides and the formation of ynones from alkynes and acyl chlorides. Furthermore, the methodology was applied to the synthesis of anemarchalconyn, an aromatic ynone isolated from the rhizomes of Anemarrhena asphodeloides.

\section{Experimental section}

\subsection{General methods}

All reactions were conducted under a dried argon stream. The reagents were purchased from Aldrich Chemical Co, St. Louis Missouri, United States, and used without further purification unless stated otherwise. Yields refer to the chromatographically and spectroscopically $\left({ }^{1} \mathrm{H}\right.$ and $\left.{ }^{13} \mathrm{C}\right)$ homogeneous materials, unless otherwise stated. All glassware was flame-dried before use. The reactions were monitored by TLC carried out on $0.25 \mathrm{~mm}$ E. Merck silica gel plates. The developed TLC plates were visualised under a short-wave UV lamp or by heating after 
they were dipped in $\mathrm{Ce}\left(\mathrm{SO}_{4}\right)_{2}$. Flash column chromatography (FCC) was performed using silica gel (230-400) and employed a solvent polarity correlated with the TLC mobility. NMR experiments were conducted on a Varian $300 \mathrm{MHz}$ instrument in $\mathrm{CDCl}_{3}(99.9 \% \mathrm{D})$ as the solvent; the chemical shifts $(\delta)$ were referenced to $\mathrm{CDCl}_{3}\left(7.26 \mathrm{ppm}{ }^{1} \mathrm{H}, 77.00 \mathrm{ppm}{ }^{13} \mathrm{C}\right.$ ) or $\mathrm{Me}_{4} \mathrm{Si}$ $(0.00 \mathrm{ppm})$. The chemical shifts are reported with respect to the solvent peak in parts per million (ppm). Microwave irradiation experiments were performed on a Monowave 450-Anton Paar, using standard sealed microwave glass vials.

\subsection{General procedures}

4.2.1. Synthesis of complex Ph1-Phoxide 8. A solution of $\mathrm{Ph}_{2} \mathrm{PCl}(0.75 \mathrm{~mL} 4.04 \mathrm{mmol})$ in anhydrous THF $(5 \mathrm{~mL})$ was added dropwise whilst stirring to a solution of $\mathrm{PdCl}_{2}\left(\mathrm{C}_{6} \mathrm{H}_{5} \mathrm{CN}\right)_{2}$ $(0.76 \mathrm{~g}, 2 \mathrm{mmol}) 9$ in anhydrous THF $(10 \mathrm{~mL})$ placed in a Schlenk flask at room temperature. When the complete formation of the dichlorophosphane complex $\mathbf{1 0}$ was confirmed by ${ }^{31} \mathrm{P}$ NMR spectroscopy $(\delta=87 \mathrm{ppm}), 0.5 \mathrm{~mL}$ of $\mathrm{H}_{2} \mathrm{O}$ was added to the reaction mixture, which was stirred at room temperature during $48 \mathrm{~h}$. After that time, the solvent was removed under reduced pressure to give the complex $\mathbf{8}$ as a yellow crystalline powder (92\% yield). ${ }^{1} \mathrm{H}$ NMR $(300 \mathrm{MHz}$, $\left.\mathrm{CDCl}_{3}\right): \delta=7.7-7.2(\mathrm{~m}, 40 \mathrm{H}) ;{ }^{31} \mathrm{P} \mathrm{NMR}\left(121.4 \mathrm{MHz}, \mathrm{CDCl}_{3}\right): \delta=$ 78.6.

4.2.2. General procedure for the synthesis of gem-1,3enynes. A solution of alkyne 11 ( $1 \mathrm{mmol}), \mathrm{NaOAc}(2 \mathrm{mmol})$ and catalyst $8(5 \% \mathrm{mmol})$ in anhydrous toluene $(25 \mathrm{~mL})$ was heated for $18 \mathrm{~h}$ at $100^{\circ} \mathrm{C}$ in a silicon oil bath, using a condenser system. Then, the mixture was cooled to room temperature, diluted with EtOAc $(25 \mathrm{~mL})$, washed with brine $(3 \times 25 \mathrm{~mL})$, dried over magnesium sulphate, filtered and concentrated under reduced pressure. The resulting residue was purified by flash column chromatography on silica gel using hexane-EtOAc.

4.2.3. General procedure for the synthesis of aromaticalkynes. A solution of aryl halide 13 (1 mmol), DIPEA ( $2 \mathrm{mmol}$ ), $\mathrm{CuI}(5 \% \mathrm{mmol})$ and catalyst $8(5 \% \mathrm{mmol})$ in anhydrous toluene $(15 \mathrm{~mL})$ was placed in a round bottom flask and evacuated/ backfilled with $\mathrm{N}_{2}$ three times. Then, an $\mathrm{N}_{2}$-purged solution of alkyne $11(1 \mathrm{mmol})$ in anhydrous toluene $(10 \mathrm{~mL})$ was added. The reaction mixture was stirred and heated at $80{ }^{\circ} \mathrm{C}$ for $18 \mathrm{~h}$ under $\mathrm{N}_{2}$ atmosphere. After that time, the reaction mixture was allowed to cool to room temperature, diluted with EtOAc (25 $\mathrm{mL})$, washed successively with $10 \%$ citric acid solution $(2 \times 20$ $\mathrm{mL}), 10 \% \mathrm{NaHCO}_{3}$ solution $(2 \times 20 \mathrm{~mL})$ and brine $(3 \times 20 \mathrm{~mL})$, dried over magnesium sulphate, filtered and concentrated under reduced pressure. The resulting residue was purified by flash column chromatography on silica gel using hexaneEtOAc.

4.2.4. General procedure for the synthesis of ynones. A solution of acyl chloride 15 ( $1 \mathrm{mmol})$, TEA $(2 \mathrm{mmol})$, CuI $(5 \%$ $\mathrm{mmol})$ and catalyst $8(5 \% \mathrm{mmol})$ in anhydrous toluene $(15 \mathrm{~mL})$ was placed in a round bottom flask and evacuated/backfilled with $\mathrm{N}_{2}$ three times. Then, an $\mathrm{N}_{2}$-purged solution of alkyne $\mathbf{1 1}$ $(1 \mathrm{mmol})$ in anhydrous toluene $(10 \mathrm{~mL})$ was added. The reaction mixture was stirred and heated at $80{ }^{\circ} \mathrm{C}$ for $18 \mathrm{~h}$ under $\mathrm{N}_{2}$ atmosphere. After that time, the reaction mixture was allowed to cool to room temperature, before being quenched with saturated aqueous ammonium chloride solution $(15 \mathrm{~mL})$. The mixture was extracted with ethyl acetate $(2 \times 15 \mathrm{~mL})$. The combined organic layers were washed successively with $10 \%$ citric acid solution $(2 \times 20 \mathrm{~mL}), 10 \% \mathrm{NaHCO}_{3}$ solution $(2 \times 20$ $\mathrm{mL})$ and brine $(3 \times 20 \mathrm{~mL})$, dried over magnesium sulphate, filtered and concentrated under reduced pressure. The resulting residue was purified by flash column chromatography on silica gel using hexane-EtOAc.

\subsection{Characterization data}

4.3.1. $\quad 1^{2 a^{28}}$ (92\% yield). ${ }^{1} \mathrm{H}$ NMR ( $\left.300 \mathrm{MHz}, \mathrm{CDCl}_{3}\right): \delta 5.20$ $(\mathrm{d}, J=1.8 \mathrm{~Hz}, 1 \mathrm{H}), 5.12(\mathrm{~d}, J=1.8 \mathrm{~Hz}, 1 \mathrm{H}), 2.29(\mathrm{t}, J=6.9 \mathrm{~Hz}$, $2 \mathrm{H}), 2.21(\mathrm{t}, J=7.2 \mathrm{~Hz}, 2 \mathrm{H}), 1.59-1.48(\mathrm{~m}, 4 \mathrm{H}), 1.36-1.29(\mathrm{~m}$, $8 \mathrm{H}), 0.90(\mathrm{t}, J=7.2 \mathrm{~Hz}, 3 \mathrm{H}), 0.89(\mathrm{t}, J=7.2 \mathrm{~Hz}, 3 \mathrm{H}) ;{ }^{13} \mathrm{C} \mathrm{NMR}(75$ $\left.\mathrm{MHz}, \mathrm{CDCl}_{3}\right): \delta 132.4,119.3,90.1,81,37.5,31.1,31,28.5,27.8$, $22.5,22.2,19.3$, 14. Anal. calcd for $\mathrm{C}_{14} \mathrm{H}_{24}$ : C, 87.42; H, 12.58 . Found: C, 87.40; H, 12.50 .

4.3.2. $12 \mathrm{~b}^{28}$ (91\% yield). ${ }^{1} \mathrm{H}$ NMR ( $\left.300 \mathrm{MHz}, \mathrm{CDCl}_{3}\right): \delta 5.20$ (d, $J=1.8 \mathrm{~Hz}, 1 \mathrm{H}), 5.12$ (d, $J=1.8 \mathrm{~Hz}, 1 \mathrm{H}), 2.29$ (t, $J=6.9 \mathrm{~Hz}$, $2 \mathrm{H}), 2.21(\mathrm{t}, J=7.2 \mathrm{~Hz}, 2 \mathrm{H}), 1.59-1.48(\mathrm{~m}, 4 \mathrm{H}), 1.36-1.29(\mathrm{~m}$, $12 \mathrm{H}), 0.90(\mathrm{t}, J=7.2 \mathrm{~Hz}, 3 \mathrm{H}), 0.89(\mathrm{t}, J=7.2 \mathrm{~Hz}, 3 \mathrm{H}) ;{ }^{13} \mathrm{C}$ NMR (75 MHz, $\left.\mathrm{CDCl}_{3}\right): \delta 132.4,119.3,90.1,81,37.5,31.1,31,29.1$, 28.8, 28.1, 27.7, 22.5, 22.3, 19.3, 14. Anal. calcd for $\mathrm{C}_{16} \mathrm{H}_{28}$ : C, 87.19; H, 12.81. Found: C, 87.16; H, 12.79.

4.3.3. $\quad$ 12c $^{29}(77 \%$ yield $) . ~{ }^{1} \mathrm{H}$ NMR $\left(300 \mathrm{MHz}, \mathrm{CDCl}_{3}\right): \delta 7.75-$ $7.70(\mathrm{~m}, 2 \mathrm{H}), 7.56-7.53(\mathrm{~m}, 2 \mathrm{H}), 7.42-7.32(\mathrm{~m}, 6 \mathrm{H}), 6.01(\mathrm{~d}, J=$ $1.0 \mathrm{~Hz}, 1 \mathrm{H}), 5.79(\mathrm{~d}, J=1.0 \mathrm{~Hz}, 1 \mathrm{H}) ;{ }^{13} \mathrm{C}$ NMR $(75.5 \mathrm{MHz}$, $\left.\mathrm{CDCl}_{3}\right): \delta 137.3,131.7,130.6,128.4,128.3,126.1,123.1,120.6$, 90.8, 88.6. Anal. calcd for $\mathrm{C}_{16} \mathrm{H}_{12}: \mathrm{C}, 94.08 ; \mathrm{H}, 5.92$. Found: $\mathrm{C}$, 94.01; H, 5.88.

4.3.4. $\quad$ 12d $^{30}$ (85\% yield). ${ }^{1} \mathrm{H}$ NMR $\left(300 \mathrm{MHz}, \mathrm{CDCl}_{3}\right): \delta 7.61$ $(\mathrm{d}, J=8.0 \mathrm{~Hz}, 2 \mathrm{H}), 7.42(\mathrm{~d}, J=8.0 \mathrm{~Hz}, 2 \mathrm{H}), 7.17(\mathrm{~d}, J=8.4 \mathrm{~Hz}$, $2 \mathrm{H}), 7.15(\mathrm{~d}, J=8.4 \mathrm{~Hz}, 2 \mathrm{H}), 5.91(\mathrm{~d}, J=1.0 \mathrm{~Hz}, 1 \mathrm{H}), 5.68(\mathrm{~d}, J=$ $0.9 \mathrm{~Hz}, 1 \mathrm{H}), 2.36(\mathrm{~s}, 6 \mathrm{H}) ;{ }^{13} \mathrm{C} \mathrm{NMR}\left(75 \mathrm{MHz}, \mathrm{CDCl}_{3}\right): \delta 138.4$, 138.1, 134.5, 131.5, 130.5, 129.1, 129, 125.9, 120, 119.4, 90.7, 88, 21.4, 21. Anal. calcd for $\mathrm{C}_{18} \mathrm{H}_{16}: \mathrm{C}, 93.06$; $\mathrm{H}, 6.94$. Found: $\mathrm{C}$, 93.01; H, 6.90.

4.3.5. $12 \mathrm{e}^{30}$ (92\% yield). ${ }^{1} \mathrm{H}$ NMR $\left(300 \mathrm{MHz}, \mathrm{CDCl}_{3}\right): \delta 7.39$ $(\mathrm{dd}, J=7.6,2.0 \mathrm{~Hz}, 1 \mathrm{H}), 7.33(\mathrm{dd}, J=7.6,2.0 \mathrm{~Hz}, 1 \mathrm{H}), 7.23-7.17$ $(\mathrm{m}, 5 \mathrm{H}), 7.13-7.09(\mathrm{~m}, 1 \mathrm{H}), 5.86(\mathrm{~d}, J=2.0 \mathrm{~Hz}, 1 \mathrm{H}), 5.53(\mathrm{~d}, J=$ $2.0 \mathrm{~Hz}, 1 \mathrm{H}), 2.49$ (s, 3H), $2.41(\mathrm{~s}, 3 \mathrm{H}) ;{ }^{13} \mathrm{C}$ NMR $\left(75 \mathrm{MHz}, \mathrm{CDCl}_{3}\right)$ : $\delta 140.1,135.5,132,131.8,130.4,129.4,128.8,128.3,127.9$, $125.9,125.5,125,123,93.3,89.6,20.7,20.3$. Anal. calcd for $\mathrm{C}_{18} \mathrm{H}_{16}$ : C, 93.06; H, 6.94. Found: C, 93.02; H, 6.91.

4.3.6. $\quad \mathbf{1 2 f}^{30}$ (83\% yield). ${ }^{1} \mathrm{H}$ NMR $\left(300 \mathrm{MHz}, \mathrm{CDCl}_{3}\right): \delta 7.52-$ $7.51(\mathrm{~m}, 2 \mathrm{H}), 7.35-7.32(\mathrm{~m}, 2 \mathrm{H}), 7.28-7.21(\mathrm{~m}, 2 \mathrm{H}), 7.15-7.13$ $(\mathrm{m}, 2 \mathrm{H}), 5.95(\mathrm{~d}, J=1.0 \mathrm{~Hz}, 1 \mathrm{H}), 5.73(\mathrm{~d}, J=1.0 \mathrm{~Hz}, 1 \mathrm{H}), 2.38(\mathrm{~s}$, $3 \mathrm{H}), 2.34(\mathrm{~s}, 3 \mathrm{H}) ;{ }^{13} \mathrm{C}$ NMR $\left(75 \mathrm{MHz}, \mathrm{CDCl}_{3}\right): \delta 138,137.9,137.3$, 132.2, 130.7, 129.3, 129.1, 128.7, 128.3, 128.2, 126.8, 123.3, 123, 120.4, 90.8, 88.4, 21.5, 21.2. Anal. calcd for $\mathrm{C}_{18} \mathrm{H}_{16}: \mathrm{C}, 93.06 ; \mathrm{H}$, 6.94. Found: C, 93.03; H, 6.92.

4.3.7. ${ }^{12 \mathrm{~g}^{30}}$ (81\% yield). ${ }^{1} \mathrm{H}$ NMR ( $\left.300 \mathrm{MHz}, \mathrm{CDCl}_{3}\right): \delta 7.71$ (d, $J=7.6 \mathrm{~Hz}, 1 \mathrm{H}), 7.43(\mathrm{~d}, J=7.6 \mathrm{~Hz}, 1 \mathrm{H}), 7.29-7.25(\mathrm{~m}, 2 \mathrm{H})$, 6.99-6.85 (m, 4H), 6.09 (brs, $1 \mathrm{H}), 5.95(\mathrm{brs}, 1 \mathrm{H}), 3.88(\mathrm{~s}, 6 \mathrm{H}) ;{ }^{13} \mathrm{C}$ 
NMR (75 MHz, $\left.\mathrm{CDCl}_{3}\right): \delta 160,157.2,133.4,130.5,129.5,129$, 128, 127.1, 125.4, 120.5, 120.4, 112.7, 111.4, 110.7, 94.1, 85.2, 55.8, 55.6. Anal. calcd for $\mathrm{C}_{18} \mathrm{H}_{16} \mathrm{O}_{2}: \mathrm{C}, 81.79 ; \mathrm{H}, 6.10$. Found: $\mathrm{C}$, 81.76; H, 6.08 .

4.3.8. $\quad$ 12h $^{30}$ (85\% yield). ${ }^{1} \mathrm{H}$ NMR (300 $\left.\mathrm{MHz}, \mathrm{CDCl}_{3}\right): \delta 7.65$ $(\mathrm{d}, J=8.4 \mathrm{~Hz}, 2 \mathrm{H}), 7.46(\mathrm{~d}, J=8.8 \mathrm{~Hz}, 2 \mathrm{H}), 6.89(\mathrm{~d}, J=8.4 \mathrm{~Hz}$, $2 \mathrm{H}), 6.86(\mathrm{~d}, J=8.8 \mathrm{~Hz}, 2 \mathrm{H}), 5.83(\mathrm{~d}, J=0.7 \mathrm{~Hz}, 1 \mathrm{H}), 5.61(\mathrm{~d}, J=$ $0.7 \mathrm{~Hz}, 1 \mathrm{H}), 3.82(\mathrm{~s}, 3 \mathrm{H}), 3.81(\mathrm{~s}, 3 \mathrm{H}) ;{ }^{13} \mathrm{C} \mathrm{NMR}$ (75 MHz, $\left.\mathrm{CDCl}_{3}\right)$ : $\delta 159.7,159.6,133.1,130.1,127.3,118.1,115.3,114,113.7,90.5$, 87.5, 55.3, 55.2. Anal. calcd for $\mathrm{C}_{18} \mathrm{H}_{16} \mathrm{O}_{2}$ : C, 81.79; H, 6.10. Found: C, 81.75; H, 6.07.

4.3.9. $12 \mathbf{i}^{30}$ (82\% yield). ${ }^{1} \mathrm{H}$ NMR (300 $\left.\mathrm{MHz}, \mathrm{CDCl}_{3}\right): \delta 7.64$ $(\mathrm{d}, J=8.4 \mathrm{~Hz}, 2 \mathrm{H}), 7.45(\mathrm{~d}, J=8.4 \mathrm{~Hz}, 2 \mathrm{H}), 6.88(\mathrm{~d}, J=8.4 \mathrm{~Hz}$, $2 \mathrm{H}), 6.85$ (d, $J=8.4 \mathrm{~Hz}, 2 \mathrm{H}), 5.82$ (brs, 1H), 5.60 (brs, 1H), 4.07$4.01(\mathrm{~m}, 4 \mathrm{H}), 1.41(\mathrm{t}, J=6.4 \mathrm{~Hz}, 6 \mathrm{H}) ;{ }^{13} \mathrm{C} \mathrm{NMR}\left(75 \mathrm{MHz}, \mathrm{CDCl}_{3}\right)$ : $\delta$ 159.1, 159, 133.1, 130.1, 129.9, 127.3, 117.9, 115.1, 114.5, 114.2, 90.6, 87.5, 63.5, 63.4, 14.8, 14.7 Anal. calcd for $\mathrm{C}_{20} \mathrm{H}_{20} \mathrm{O}_{2}$ : C, 82.16; H, 6.89. Found: C, 82.14; H, 6.86.

4.3.10. $12 \mathbf{j}^{30}$ (76\% yield). ${ }^{1} \mathrm{H}$ NMR $\left(300 \mathrm{MHz}, \mathrm{CDCl}_{3}\right): \delta 7.65$ $(\mathrm{d}, J=8.4 \mathrm{~Hz}, 2 \mathrm{H}), 7.46-7.32(\mathrm{~m}, 12 \mathrm{H}), 6.96(\mathrm{~d}, J=8.4 \mathrm{~Hz}, 2 \mathrm{H})$, 6.94 (d, $J=8.8 \mathrm{~Hz}, 2 \mathrm{H}$ ), 5.83 (brs, 1H), 5.61 (brs, 1H), 5.08 (brs, 2H), 5.07 (brs, 2H); ${ }^{13} \mathrm{C} \mathrm{NMR} \mathrm{(75} \mathrm{MHz}, \mathrm{CDCl}_{3}$ ): $\delta$ 158.9, 158, 136.9, 136.6, 133.1, 130.3, 130, 128.6, 128.6, 128.1, 128, 127.5, 127.4, 127.3, 118.2, 115.5, 114.9, 114.6, 90.5, 87.6, 70, 70; Anal. calcd for $\mathrm{C}_{30} \mathrm{H}_{24} \mathrm{O}_{2}$ : C, 86.51; H, 5.81. Found: C, 86.46; H, 5.77.

4.3.11. $14 \mathrm{a}^{31}(82 \%$ for bromo- and $91 \%$ for iodo-substituted aryls). ${ }^{1} \mathrm{H}$ NMR $\left(300 \mathrm{MHz}, \mathrm{CDCl}_{3}\right): \delta 7.90(\mathrm{dd}, J=8.3,1.2 \mathrm{~Hz}$, $1 \mathrm{H}), 7.51$ (dd, $J=7.8,1.6 \mathrm{~Hz}, 1 \mathrm{H}), 7.46$ (ddd, $J=7.8,7.7,1.2 \mathrm{~Hz}$, $1 \mathrm{H}), 7.33$ (ddd, $J=8.3,7.7,1.6 \mathrm{~Hz}, 1 \mathrm{H}), 2.42$ (t, $J=7.2 \mathrm{~Hz}, 2 \mathrm{H}$ ), 1.63-1.56 (m, 2H), 1.45-1.28 (m, 4H), $0.89(\mathrm{t}, J=7.2 \mathrm{~Hz}, 3 \mathrm{H}) ;{ }^{13} \mathrm{C}$ NMR (75 MHz, $\left.\mathrm{CDCl}_{3}\right): \delta 150,134.6,132.3,127.7,124.2,119.3$, 99.2 , 75.9, 30.9, 27.9, 22.1, 19.7, 13.9. Anal. calcd for $\mathrm{C}_{13} \mathrm{H}_{15} \mathrm{NO}_{2}$ : C, 71.87; H, 6.96; N, 6.45. Found: C, 71.82; H, 6.91; N, 6.39.

4.3.12. $14 b^{32}(83 \%$ for bromo- and $92 \%$ for iodosubstituted aryls). ${ }^{1} \mathrm{H} \mathrm{NMR}\left(300 \mathrm{MHz}, \mathrm{CDCl}_{3}\right): \delta 7.43-7.41(\mathrm{~m}$, $2 \mathrm{H}), 7.30-7.29(\mathrm{~m}, 3 \mathrm{H}), 2.43(\mathrm{t}, J=7.2 \mathrm{~Hz}, 2 \mathrm{H}), 1.67-1.60(\mathrm{~m}$, $2 \mathrm{H}), 1.48-1.37(\mathrm{~m}, 4 \mathrm{H}), 0.95(\mathrm{t}, J=7.2 \mathrm{~Hz}, 3 \mathrm{H}) ;{ }^{13} \mathrm{C} \mathrm{NMR}(75$ $\left.\mathrm{MHz}, \mathrm{CDCl}_{3}\right): \delta$ ppm 133.1, 129.8, 129.1, 125.6, 92.1, 82.1, 32.7, 30.1 , 23.9, 21, 15.7; anal. calcd for $\mathrm{C}_{13} \mathrm{H}_{16}$ : C, 90.64; H, 9.36. Found: C, 90.61; H, 9.32.

4.3.13. $14 \mathrm{c}^{33}(81 \%$ for bromo- and $90 \%$ for iodo-substituted aryls). ${ }^{1} \mathrm{H}$ NMR $\left(300 \mathrm{MHz}, \mathrm{CDCl}_{3}\right): \delta 7.99(\mathrm{dd}, J=8.2,1.4 \mathrm{~Hz}$, $1 \mathrm{H}), 7.64$ (dd, $J=7.8,1.4 \mathrm{~Hz}, 1 \mathrm{H}), 7.54(\mathrm{ddd}, J=7.8,7.8,1.4 \mathrm{~Hz}$, 1H), 7.46-7.41 (m, 1H), 0.27 (s, 9H); $\left.{ }^{13} \mathrm{C} \mathrm{NMR} \mathrm{(75} \mathrm{MHz,} \mathrm{CDCl}_{3}\right)$ : $\delta$ ppm 150.3, 135.2, 132.7, 128.9, 124.5, 118.5, 103.8, 99.4, -0.3; anal. calcd for $\mathrm{C}_{11} \mathrm{H}_{13} \mathrm{NO}_{2} \mathrm{Si}$ : C, 60.24; $\mathrm{H}, 5.97 ; \mathrm{N}, 6.39$. Found: C, $60.21 ; \mathrm{H}, 5.95 ; \mathrm{N}, 6.37$.

4.3.14. $14 d^{32} \quad(80 \%$ for bromo- and $92 \%$ for iodosubstituted aryls). ${ }^{1} \mathrm{H}$ NMR $\left(300 \mathrm{MHz}, \mathrm{CDCl}_{3}\right): \delta 7.53(\mathrm{~d}, J=$ 7.6, 2H), 7.46 (d, $J=8.4 \mathrm{~Hz}, 2 \mathrm{H}), 7.36-7.32(\mathrm{~m}, 5 \mathrm{H}) ;{ }^{13} \mathrm{C} \mathrm{NMR}(75$ $\left.\mathrm{MHz}, \mathrm{CDCl}_{3}\right): \delta 135.9,134.5,133.3,130.3,130.1,130,124.6$, 123.4, 91.9, 89.9; anal. calcd for $\mathrm{C}_{14} \mathrm{H}_{9} \mathrm{Cl}$ : C, 79.06; $\mathrm{H}, 4.27$. Found: C, 79.02; H, 4.23.

4.3.15. $14 \mathrm{e}^{32}(81 \%$ for bromo- and $90 \%$ for iodo-substituted aryls). ${ }^{1} \mathrm{H} \mathrm{NMR}\left(300 \mathrm{MHz}, \mathrm{CDCl}_{3}\right): \delta 7.52-7.50(\mathrm{~m}, 2 \mathrm{H}), 7.47(\mathrm{~d}, J$ $=8.8 \mathrm{~Hz}, 2 \mathrm{H}), 7.36-7.32(\mathrm{~m}, 3 \mathrm{H}), 6.88(\mathrm{~d}, J=8.8 \mathrm{~Hz}, 2 \mathrm{H}), 3.83(\mathrm{~s}$, $3 \mathrm{H}) ;{ }^{13} \mathrm{C} \mathrm{NMR}\left(75 \mathrm{MHz} \mathrm{CDCl}_{3}\right): \delta 161.2,134.7,133.1,130,129.6$,
125.2, 117, 115.6, 91, 89.7, 56.9; anal. calcd for $\mathrm{C}_{15} \mathrm{H}_{12} \mathrm{O}$ : C, 86.51; H, 5.81. Found: C, 86.48; H, 5.77.

4.3.16. $14 \mathrm{f}^{34}(80 \%$ for bromo- and $91 \%$ for iodo-substituted aryls). ${ }^{1} \mathrm{H}$ NMR (300 MHz, $\left.\mathrm{CDCl}_{3}\right): \delta 7.59-7.55(\mathrm{~m}, 2 \mathrm{H}), 7.43-7.37$ (m, 4H), 7.21-7.15 (m, 1H), 6.79-6.75 (m, 2H), 4.29 (brs, $2 \mathrm{H}) ;{ }^{13} \mathrm{C}$ $\mathrm{NMR}\left(75 \mathrm{MHz}, \mathrm{CDCl}_{3}\right): \delta 148.1,132.6,131.9,130.2,128.8,128.6$, $123.7,118.5,114.8,108.4,95.1,86.3$; anal. calcd for $\mathrm{C}_{14} \mathrm{H}_{11} \mathrm{~N}$ : C, 87.01; H, 5.74; N, 7.25. Found: C, 86.99; H, 5.72; N, 7.23.

4.3.17. $14 \mathrm{~g}^{35}(83 \%$ for bromo- and $91 \%$ for iodo-substituted aryls). ${ }^{1} \mathrm{H} \mathrm{NMR}\left(300 \mathrm{MHz}, \mathrm{CDCl}_{3}\right): \delta 8.59$ (brd, $\left.J=4.1 \mathrm{~Hz}, 1 \mathrm{H}\right)$; 7.64 (ddd, $J=7.7,7.7,1.8 \mathrm{~Hz}, 1 \mathrm{H}), 7.60-7.54(\mathrm{~m}, 2 \mathrm{H}), 7.50$ (d, $J=$ $7.8 \mathrm{~Hz}, 1 \mathrm{H}), 7.37-7.30$ (m, 3H), 7.23-7.17 (m, 1H); ${ }^{13} \mathrm{C}$ NMR (75 $\left.\mathrm{MHz}, \mathrm{CDCl}_{3}\right): \delta 149.7,143.1,135.9,131.7,128.7,128.1,126.9$, 122.5, 122, 89, 88.4. Anal. calcd for $\mathrm{C}_{13} \mathrm{H}_{9} \mathrm{~N}$ : C, 87.12; $\mathrm{H}, 5.06 ; \mathrm{N}$, 7.82. Found: C, 87.08; H, 5.03; N, 7.80.

4.3.18. $14 \mathrm{~h}^{36}(80 \%$ for bromo- and $90 \%$ for iodosubstituted aryls). ${ }^{1} \mathrm{H}$ NMR $\left(300 \mathrm{MHz}, \mathrm{CDCl}_{3}\right): \delta 8.77$ (brs, $1 \mathrm{H}), 8.55(\mathrm{dd}, J=6.4,1.4 \mathrm{~Hz}, 1 \mathrm{H}), 7.80(\mathrm{td}, J=8.0,2.4 \mathrm{~Hz}, 1 \mathrm{H})$, 7.56-7.54 (m, 2H), 7.38-7.36 (m, 3H), 7.30-7.26 (m, $1 \mathrm{H}) ;{ }^{13} \mathrm{C}$ NMR (75 MHz, $\mathrm{CDCl}_{3}$ ): $\delta 152.1,148.4,138.3,131.6,128.7,128.3$, 122.9, 122.4, 120.3, 92.5, 85.8, anal. calcd for $\mathrm{C}_{13} \mathrm{H}_{9} \mathrm{~N}$ : C, 87.12; H, 5.06; N, 7.82. Found: C, 87.09; H, 5.04; N, 7.80.

4.3.19. $14 \mathrm{i}^{37}(80 \%$ for bromo- and $92 \%$ for iodo-substituted aryls). ${ }^{1} \mathrm{H} \mathrm{NMR}\left(300 \mathrm{MHz}, \mathrm{CDCl}_{3}\right): \delta 7.52-7.50(\mathrm{~m}, 2 \mathrm{H}), 7.35-7.32$ (m, 3H), 7.29-7.28 (m, 2H), 7.01 (dd, $J=5.0,3.6 \mathrm{~Hz}, 1 \mathrm{H}) ;{ }^{13} \mathrm{C}$ NMR (75 MHz, $\mathrm{CDCl}_{3}$ ): $\delta$ 132.3, 131.8, 128.8, 128.7, 127.6, 127.5, 123.7, 123.3, 93.4, 83. Anal. calcd for $\mathrm{C}_{12} \mathrm{H}_{8} \mathrm{~S}: \mathrm{C}, 78.22 ; \mathrm{H}, 4.38 ; \mathrm{S}$, 17.40. Found: C, 78.20; H, 4.35; S, 17.38 .

4.3.20. $\quad$ 16a $^{38}$ (93\% yield). ${ }^{1} \mathrm{H}$ NMR (300 $\left.\mathrm{MHz} \mathrm{CDCl}_{3}\right): \delta 8.13$ (d, $J=8.25 \mathrm{~Hz}, 2 \mathrm{H}), 7.59(\mathrm{td}, J=7.6,1.1 \mathrm{~Hz}, 1 \mathrm{H}), 7.4(\mathrm{~m}, 2 \mathrm{H})$, $2.49(\mathrm{t}, J=7.1 \mathrm{~Hz}, 2 \mathrm{H}), 1.68(\mathrm{~m}, 2 \mathrm{H}), 1.34-1.48(\mathrm{~m}, 4 \mathrm{H}), 0.93(\mathrm{t}, J$ $=7.2 \mathrm{~Hz}, 3 \mathrm{H}) ;{ }^{13} \mathrm{C} \mathrm{NMR}\left(75 \mathrm{MHz}, \mathrm{CDCl}_{3}\right): \delta 178.2,136.9,133.8$, $129.5,128.4$, 96.8, 79.6, 31, 27.4, 22, 19.1, 13.8. Anal. calcd for $\mathrm{C}_{14} \mathrm{H}_{16} \mathrm{O}: \mathrm{C}, 83.96 ; \mathrm{H}, 8.05$. Found: C, 83.94; H, 8.02.

4.3.21. $16 \mathbf{b}^{39}$ (81\% yield). ${ }^{1} \mathrm{H}$ NMR $\left(300 \mathrm{MHz}, \mathrm{CDCl}_{3}\right): \delta 8.08$ $(\mathrm{d}, J=8.6 \mathrm{~Hz}, 2 \mathrm{H}), 7.45(\mathrm{~d}, J=8.6 \mathrm{~Hz}, 2 \mathrm{H}), 2.50(\mathrm{t}, J=7.1 \mathrm{~Hz}$, $2 \mathrm{H}), 1.68(\mathrm{~m}, 2 \mathrm{H}), 1.34-1.47(\mathrm{~m}, 4 \mathrm{H}), 0.93(\mathrm{t}, J=7.2 \mathrm{~Hz}, 3 \mathrm{H}) ;{ }^{13} \mathrm{C}$ NMR (75 MHz, $\mathrm{CDCl}_{3}$ ): $\delta 177,140.5,135.5,131,128.9,97.6,79.5$, 31.2 27.6, 22.2, 19.3, 14. Anal. calcd for $\mathrm{C}_{14} \mathrm{H}_{15} \mathrm{ClO}: \mathrm{C}, 71.4 ; \mathrm{H}$, 6.44. Found: C, 71.3; H, 6.42.

4.3.22. $\quad{ }^{16 c^{39}}$ (82\% yield). ${ }^{1} \mathrm{H}$ NMR (300 $\left.\mathrm{MHz} \mathrm{CDCl}_{3}\right): \delta 8.10$ $(\mathrm{d}, J=8.9 \mathrm{~Hz}, 2 \mathrm{H}), 6.94(\mathrm{~d}, J=8.9 \mathrm{~Hz}, 2 \mathrm{H}), 3.88(\mathrm{~s}, 3 \mathrm{H}), 2.48(\mathrm{t}, J$ $=7.1 \mathrm{~Hz}, 2 \mathrm{H}), 1.67(\mathrm{~m}, 2 \mathrm{H}), 1.34-1.47(\mathrm{~m}, 4 \mathrm{H}), 0.93(\mathrm{t}, J=$ $7.2 \mathrm{~Hz}, 3 \mathrm{H}) ;{ }^{13} \mathrm{C}$ NMR $\left(75 \mathrm{MHz}, \mathrm{CDCl}_{3}\right): \delta 177,164.4,132,130.4$, 113.8, 95.1, 79.7, 55.6, 31.2, 27.6, 22.2, 19.2, 14. Anal. calcd for $\mathrm{C}_{15} \mathrm{H}_{18} \mathrm{O}_{2}$ : C, 78.23; H, 7.88. Found: C, 78.21; H, 7.87.

4.3.23. $\quad$ 16d $^{38}$ (85\% yield). ${ }^{1} \mathrm{H}$ NMR (300 $\left.\mathrm{MHz} \mathrm{CDCl}_{3}\right): \delta 8.19$ (dd, $J=8.0,1.6 \mathrm{~Hz}, 2 \mathrm{H}), 7.63(\mathrm{~d}, J=8.0 \mathrm{~Hz}, 2 \mathrm{H}), 7.57(\mathrm{t}, J=$ $8.0 \mathrm{~Hz}, 1 \mathrm{H}), 7.48-7.34(\mathrm{~m}, 5 \mathrm{H}) ;{ }^{13} \mathrm{C} \mathrm{NMR}\left(75 \mathrm{MHz}, \mathrm{CDCl}_{3}\right.$ ): $\delta 178.2,137,134.4,133.3,131.1,129.8,129,128.9,120.2$, 93.4, 87.2. Anal. calcd for $\mathrm{C}_{15} \mathrm{H}_{10} \mathrm{O}: \mathrm{C}, 87.36 ; \mathrm{H}, 4.84$. Found: $\mathrm{C}, 87.35$; $\mathrm{H}, 4.83$.

4.3.24. $16 \mathrm{e}^{38}$ (82\% yield). ${ }^{1} \mathrm{H}$ NMR (300 $\left.\mathrm{MHz}, \mathrm{CDCl}_{3}\right): \delta 8.09$ $(\mathrm{d}, J=8.8 \mathrm{~Hz}, 2 \mathrm{H}), 7.56(\mathrm{~d}, J=7.6 \mathrm{~Hz}, 2 \mathrm{H}), 7.32-7.28(\mathrm{~m}, 3 \mathrm{H})$, $6.87(\mathrm{~d}, J=8.8 \mathrm{~Hz}, 2 \mathrm{H}), 3.76(\mathrm{~s}, 3 \mathrm{H}) ;{ }^{13} \mathrm{C} \mathrm{NMR}\left(75 \mathrm{MHz}, \mathrm{CDCl}_{3}\right)$ : $\delta 176.6,164.5,132.9,131.9,130.6,130.2,128.6,120.2,113.8$, 
92.3, 86.9, 55.5. Anal. calcd for $\mathrm{C}_{16} \mathrm{H}_{12} \mathrm{O}_{2}$ : C, 81.34; $\mathrm{H}, 5.12$. Found: C, 81.32; H, 5.11.

4.3.25. $\quad \mathbf{1 6 f}^{\mathbf{3 8}}$ (80\% yield). ${ }^{1} \mathrm{H}$ NMR $\left(300 \mathrm{MHz}, \mathrm{CDCl}_{3}\right): \delta 8.07$ (ddd, $J=7.8,1.8,0.3 \mathrm{~Hz}, 1 \mathrm{H}), 7.65-7.61(\mathrm{~m}, 2 \mathrm{H}), 7.57-7.51(\mathrm{~m}$, 1H), 7.48-7.36 (m, 3H), 7.08-7.00 (m, 2H), 3.04 (s, 3H); ${ }^{13} \mathrm{C}$ NMR $\left(75 \mathrm{MHz}_{\mathrm{CDCl}}\right)$ : $\delta$ 176.7, 159.8, 134.9, 132.9, 132.5, 130.4, 128.5, 126.7, 120.6, 120.2, 112.1, 91.5, 89.1, 55.9. Anal. calcd for $\mathrm{C}_{16} \mathrm{H}_{12} \mathrm{O}_{2}$ : C, 81.34; H, 5.12. Found: C, 81.31; H, 5.09.

4.3.26. $16 \mathbf{g}^{38}$ (83\% yield). ${ }^{1} \mathrm{H}$ NMR $\left(300 \mathrm{MHz}, \mathrm{CDCl}_{3}\right): \delta 8.16$ (d, $J=8.8 \mathrm{~Hz}, 2 \mathrm{H}), 7.70$ (d, $J=7.9 \mathrm{~Hz}, 2 \mathrm{H}), 7.51-7.40(\mathrm{~m}, 5 \mathrm{H})$; ${ }^{13} \mathrm{C}$ NMR (75 MHz, $\mathrm{CDCl}_{3}$ ): $\delta$ 176.4, 140.6, 135.3, 132.9, 130.8, 130.7, 128.8, 128.6, 119.8, 93.5, 86.5. Anal. calcd for $\mathrm{C}_{15} \mathrm{H}_{9} \mathrm{ClO}$ : C, 74.85; H, 3.77. Found: C, 74.86; H, 3.78.

4.3.27. $16 \mathrm{~h}^{40}$ (80\% yield). ${ }^{1} \mathrm{H}$ NMR $\left(\mathrm{CDCl}_{3}, 300 \mathrm{MHz}\right)$ : $\delta$ 7.71-7.64 (m, 3H), 7.48-7.39 (m, $4 \mathrm{H}), 6.62(\mathrm{dd}, J=3.8,1.8 \mathrm{~Hz}$, $1 \mathrm{H}) ;{ }^{13} \mathrm{C} \mathrm{NMR}\left(\mathrm{CDCl}_{3}, 75.5 \mathrm{MHz}\right): \delta 164.7,153.2,147.9,132.9$, $130.8,128.6,120.8,119.9,112.6,91.8,86.2$. Anal. calcd for $\mathrm{C}_{13} \mathrm{H}_{8} \mathrm{O}_{2}$ : C, 79.58; H, 4.11. Found: C, 79.55; H, 4.8.

4.3.28. $16 \mathbf{1}^{\mathbf{3 9}}$ (80\% yield). ${ }^{1} \mathrm{H}$ NMR $\left(300 \mathrm{MHz} \mathrm{CDCl}_{3}\right): \delta 7.88$ $(\mathrm{dd}, J=3.8,1.0 \mathrm{~Hz}, 1 \mathrm{H}), 7.67(\mathrm{dd}, J=4.9,1.0 \mathrm{~Hz}, 1 \mathrm{H}), 7.14(\mathrm{t}, J=$ $3.9 \mathrm{~Hz}, 1 \mathrm{H}), 2.46(\mathrm{t}, J=7.1 \mathrm{~Hz}, 2 \mathrm{H}), 1.66(\mathrm{~m}, 2 \mathrm{H}), 1.46-1.33(\mathrm{~m}$, $4 \mathrm{H}), 0.92(\mathrm{t}, J=7.2 \mathrm{~Hz}, 3 \mathrm{H}) ;{ }^{13} \mathrm{C} \mathrm{NMR}\left(75.5 \mathrm{MHz}, \mathrm{CDCl}_{3}\right.$ ): $\delta$ 170.2, 145.2, 135, 128.3, 95.6, 79.4, 31.2, 27.5, 22.2, 19.2, 14. Anal. calcd for $\mathrm{C}_{12} \mathrm{H}_{14} \mathrm{OS}$ : C, 69.86; H, 6.84; S, 15.54. Found: C, 69.83; H, 6.81; S, 15.52.

\subsection{Synthesis of anemarchalconyn}

4.4.1. 1-Iodo-4-methoxybenzene 21. $p$-Iodophenol 20 (1 g, $4.5 \mathrm{mmol})$ and $\mathrm{NaOH}(800 \mathrm{mg}, 20 \mathrm{mmol})$ were mixed and ground in a mortar to have a fine powder. Then, the mixture was placed in a three-necked round-bottomed flask $(100 \mathrm{~mL})$ fitted with an efficient stirrer, separatory funnel and reflux condenser. The mixture was cooled at $0{ }^{\circ} \mathrm{C}$. There was then added through the separatory funnel, with stirring, dimethyl sulfate $(252 \mathrm{mg}$, $4.5 \mathrm{mmol}$ ). This addition at $0{ }^{\circ} \mathrm{C}$ requires one hour. The mixture was then heated for $18 \mathrm{~h}$ at $60^{\circ} \mathrm{C}$. After that time, the reaction mixture was allowed to cool to room temperature, before being quenched with $\mathrm{H}_{2} \mathrm{O}(25 \mathrm{~mL})$. The mixture was extracted with ethyl acetate $(2 \times 20 \mathrm{~mL})$. The combined organic layers were washed successively with $10 \%$ citric acid solution $(2 \times 20 \mathrm{~mL})$ and brine $(3 \times 20 \mathrm{~mL})$, dried over magnesium sulphate, filtered and concentrated under reduced pressure. A with solid was obtained after the recrystallization of the residue with hexaneEtOAc (947 mg, 90\% yield). ${ }^{1} \mathrm{H}$ NMR (300 MHz, $\left.\mathrm{CDCl}_{3}\right): \delta 7.55$ (d, $J=8.4 \mathrm{~Hz}, 2 \mathrm{H}), 6.68(\mathrm{~d}, J=8.4 \mathrm{~Hz}, 2 \mathrm{H}), 3.78(\mathrm{~s}, 3 \mathrm{H}) ;{ }^{13} \mathrm{C}$ NMR $(75$ $\left.\mathrm{MHz}, \mathrm{CDCl}_{3}\right): \delta 159.7,138.4,116.6,82.9,55.5$. Anal. calcd for $\mathrm{C}_{7} \mathrm{H}_{7} \mathrm{IO}: \mathrm{C}, 35.92 ; \mathrm{H}, 3.01$. Found: C, 35.91; H, 3.00.

4.4.2. ((4-Methoxyphenyl)ethynyl)trimethylsilane 22. A solution of 1-iodo-4-methoxybenzene 21 (850 $\mathrm{mg}, 3.63 \mathrm{mmol}$ ), DIPEA (480 mg, $3.72 \mathrm{mmol}$ ), CuI (34 mg, $0.18 \mathrm{mmol}, 5 \% \mathrm{mmol}$ ) and catalyst $8(196 \mathrm{mg}, 0.18 \mathrm{mmol}, 5 \% \mathrm{mmol})$ in anhydrous toluene $(20 \mathrm{~mL})$ was placed in a round bottom flask and evacuated/backfilled with $\mathrm{N}_{2}$ three times. Then, an $\mathrm{N}_{2}$-purged solution of ethynyltrimethylsilane (365 mg, $3.72 \mathrm{mmol}$ ) in anhydrous toluene $(10 \mathrm{~mL})$ was added. The reaction mixture was stirred and heated at $80{ }^{\circ} \mathrm{C}$ for $18 \mathrm{~h}$ under $\mathrm{N}_{2}$ atmosphere.
After that time, the reaction mixture was allowed to cool to room temperature, diluted with EtOAc $(25 \mathrm{~mL})$, washed successively with $10 \%$ citric acid solution $(2 \times 20 \mathrm{~mL}), 10 \% \mathrm{NaHCO}_{3}$ solution $(2 \times 20 \mathrm{~mL})$ and brine $(3 \times 20 \mathrm{~mL})$, dried over magnesium sulphate, filtered and concentrated under reduced pressure. The resulting residue was purified by flash column chromatography on silica gel using hexane-EtOAc, to give $592 \mathrm{mg}$ of 22 (80\% yield). ${ }^{1} \mathrm{H}$ NMR (300 $\left.\mathrm{MHz}, \mathrm{CDCl}_{3}\right): \delta 7.41(\mathrm{~d}, J=8.4 \mathrm{~Hz}$, $2 \mathrm{H}), 6.82(\mathrm{~d}, J=8.4 \mathrm{~Hz}, 2 \mathrm{H}), 3.81(\mathrm{~s}, 3 \mathrm{H}), 0.25(\mathrm{~s}, 9 \mathrm{H}) ;{ }^{13} \mathrm{C} \mathrm{NMR}$ (75 MHz, $\mathrm{CDCl}_{3}$ ): $\delta 159.7,133.4,115.2,113.7,105.1$, 92.4, 55.2, 0.06. Anal. calcd for $\mathrm{C}_{12} \mathrm{H}_{16} \mathrm{OSi}$ C, 70.53; H, 7.89. Found: C, $70.51 ; \mathrm{H}, 7.87$.

4.4.3. 1-Ethynyl-4-methoxybenzene $14 \mathrm{j}$. $\mathrm{K}_{2} \mathrm{CO}_{3}(45 \mathrm{mg}, 0.33$ $\mathrm{mmol})$ and the mixture $\mathrm{MeOH}-\mathrm{CHCl}_{3}(10 \mathrm{~mL}, 1: 1)$ were added to ((4-methoxyphenyl)ethynyl)trimethylsilane 22 (200 mg, 0.98 $\mathrm{mmol}$ ) in a $50 \mathrm{~mL}$ recovery flask. The suspension was stirred at room temperature for $18 \mathrm{~h}$. After that time, the reaction mixture was diluted with EtOAc $(25 \mathrm{~mL})$, washed successively with $10 \%$ citric acid solution $(2 \times 20 \mathrm{~mL})$ and brine $(3 \times 20 \mathrm{~mL})$, dried over magnesium sulphate, filtered and concentrated under reduced pressure. The resulting residue was purified by flash column chromatography on silica gel using hexane-EtOAc, to give $116 \mathrm{mg}$ of $\mathbf{1 4 j}$ (90\% yield). ${ }^{1} \mathrm{H}$ NMR $\left(300 \mathrm{MHz}, \mathrm{CDCl}_{3}\right): \delta 7.46$ $(\mathrm{d}, J=8.4 \mathrm{~Hz}, 2 \mathrm{H}), 6.87(\mathrm{~d}, J=8.4 \mathrm{~Hz}, 2 \mathrm{H}), 3.84(\mathrm{~s}, 3 \mathrm{H}), 3.02(\mathrm{~s}$, $1 \mathrm{H}) ;{ }^{13} \mathrm{C}$ NMR $\left(75.5 \mathrm{MHz}, \mathrm{CDCl}_{3}\right): \delta 159.9,133.5,114.2,113.9$, 93.9, 83.6, 55.3. Anal. calcd for $\mathrm{C}_{9} \mathrm{H}_{8} \mathrm{O}: \mathrm{C}, 81.79 ; \mathrm{H}, 6.10$. Found: C, 81.76; H, 6.08 .

4.4.4. 4-Methoxybenzoic acid 24. A solution of (4-methoxyphenyl)methanol 23 (600 $\mathrm{mg}, 4.41 \mathrm{mmol})$ in $10 \mathrm{~mL}$ of acetone was added to a $100 \mathrm{~mL}$ three-necked flask fitted with a longstem dropping funnel and a thermometer. The agitated solution was cooled in a water bath to about $20^{\circ}$. The Jones reagent $\left(\mathrm{CrO}_{3} / \mathrm{H}_{2} \mathrm{SO}_{4}\right)$ was added from the dropping funnel. The rate of addition was adjusted so that the temperature of the reaction mixture does not rise above $25^{\circ}$. After the complete conversion of starting material, monitored by thin layer chromatographic, the reaction mixture was allowed to cool to room temperature, before being quenched with isopropanol $(10 \mathrm{~mL})$. Then, the reaction mixture was filtrated to eliminate chromium salts. The resulting residue was purified by flash column chromatography on silica gel using hexane-EtOAc, to give $603 \mathrm{mg}$ of $\mathbf{2 4}(90 \%$ yield). ${ }^{1} \mathrm{H}$ NMR (300 MHz, DMSO-d $\left.{ }_{6}\right): \delta 7.90(\mathrm{~d}, J=8.4 \mathrm{~Hz}, 2 \mathrm{H})$, $7.00(\mathrm{~d}, J=8.4 \mathrm{~Hz}, 2 \mathrm{H}), 3.81(\mathrm{~s}, 3 \mathrm{H}) ;{ }^{13} \mathrm{C}$ NMR (75 MHz, DMSO$\left.\mathrm{d}_{6}\right): \delta 169.3,165.8,131.3,122.5,114.2,55.8$. Anal. calcd for $\mathrm{C}_{8} \mathrm{H}_{8} \mathrm{O}_{3}$ : C, 63.15; H, 5.30. Found: C, 63.13; H, 5.28.

4.4.5. 4-Methoxybenzoyl chloride 25. To stirred solution of carboxylic acid $24(200 \mathrm{mg}, 1.36 \mathrm{mmol})$ in toluene $(10 \mathrm{~mL})$, $\mathrm{SOCl}_{2}(810 \mathrm{mg}, 6.8 \mathrm{mmol})$ was added at room temperature. Then, the reaction mixture was stirred and heated at reflux temperature for $6 \mathrm{~h}$ under $\mathrm{N}_{2}$ atmosphere. After that time, the reaction mixture was cooled to room temperature and concentrated under reduced pressure. The resulting residue was used in next reaction without further purification.

4.4.6. 1,3-Bis(4-methoxyphenyl)prop-2-yn-1one 16j. A solution of acyl chloride 25 (150 $\mathrm{mg}, 0.87 \mathrm{mmol}$ ), CuI (8 mg, $0.043 \mathrm{mmol}, 5 \% \mathrm{mmol}$ ) and catalyst 8 (47 mg, $0.043 \mathrm{mmol}, 5 \%$ $\mathrm{mmol})$ in anhydrous TEA $(15 \mathrm{~mL})$ was placed in a round bottom 
flask and evacuated/backfilled with $\mathrm{N}_{2}$ three times. Then, an $\mathrm{N}_{2}$ purged solution of alkyne $\mathbf{1 4 j}$ (92 $\mathrm{mg}, 0.7 \mathrm{mmol}$ ) in anhydrous TEA $(10 \mathrm{~mL})$ was added. The reaction mixture was stirred and heated at $80{ }^{\circ} \mathrm{C}$ for $18 \mathrm{~h}$ under $\mathrm{N}_{2}$ atmosphere. After that time, the reaction mixture was allowed to cool to room temperature, before being quenched with saturated aqueous ammonium chloride solution $(15 \mathrm{~mL})$. The mixture was extracted with ethyl acetate $(2 \times 15 \mathrm{~mL})$. The combined organic layers were washed successively with $10 \%$ citric acid solution $(2 \times 20 \mathrm{~mL}), 10 \%$ $\mathrm{NaHCO}_{3}$ solution $(2 \times 20 \mathrm{~mL})$ and brine $(3 \times 20 \mathrm{~mL})$, dried over magnesium sulphate, filtered and concentrated under reduced pressure. The resulting residue was purified by flash column chromatography on silica gel using hexane-EtOAc. To give $149 \mathrm{mg}$ of $16 \mathrm{j}$ (80\% yield). ${ }^{1} \mathrm{H}$ NMR $\left(300 \mathrm{MHz}, \mathrm{CDCl}_{3}\right): \delta 8.19(\mathrm{~d}, J$ $=8.7 \mathrm{~Hz}, 2 \mathrm{H}), 7.63(\mathrm{~d}, J=8.7 \mathrm{~Hz}, 2 \mathrm{H}), 6.99(\mathrm{~d}, J=8.7 \mathrm{~Hz}, 2 \mathrm{H})$, $6.93(\mathrm{~d}, J=8.7 \mathrm{~Hz}, 2 \mathrm{H}), 3.90(\mathrm{~s}, 3 \mathrm{H}), 3.86$ (s, 3H); ${ }^{13} \mathrm{C}$ NMR (75 $\left.\mathrm{MHz}, \mathrm{CDCl}_{3}\right): \delta 176.7,164.3,161.6,134.9,131.9,130.5,114.4$, 113.8, 112.2, 93.4, 86.8, 55.6, 55.4. Anal. calcd for $\mathrm{C}_{17} \mathrm{H}_{14} \mathrm{O}_{3}: \mathrm{C}$, 76.68; H, 5.30. Found: C, 76.66; H, 5.28.

4.4.7. Anemarchalconyn 16k. ${ }^{27}$ A solution of $16 \mathbf{j}(145 \mathrm{mg}$, $0.54 \mathrm{mmol}$ ) in $5 \mathrm{~mL}$ of DMF was placed in a round bottom. Then, iodocyclohexane $(1.3 \mathrm{~mL}, 10.0 \mathrm{mmol})$ was added. ${ }^{41}$ The reaction system was equipped with reflux condenser under argon. The reaction mixture was stirred and heated at $90{ }^{\circ} \mathrm{C}$ for $18 \mathrm{~h}$ under $\mathrm{N}_{2}$ atmosphere. After that time, the reaction mixture was allowed to cool to room temperature, before being quenched with saturated aqueous ammonium chloride solution $(10 \mathrm{~mL})$. The mixture was extracted with ethyl acetate $(2 \times$ $10 \mathrm{~mL}$ ). The combined organic layers were washed successively with $10 \% \mathrm{NaHCO}_{3}$ solution $(2 \times 10 \mathrm{~mL})$ and brine $(2 \times 10 \mathrm{~mL})$, dried over magnesium sulphate, filtered and concentrated under reduced pressure. The resulting residue was purified by flash column chromatography on silica gel using hexaneEtOAc. To give $45 \mathrm{mg}$ of anemarchalconyn $16 \mathrm{k}$ (35\% yield). ${ }^{1} \mathrm{H}$ NMR (300 MHz, CD $\left.{ }_{3} \mathrm{OD}\right): \delta 8.07(\mathrm{~d}, J=8.7 \mathrm{~Hz}, 2 \mathrm{H}), 7.55(\mathrm{~d}, J=$ $8.7 \mathrm{~Hz}, 2 \mathrm{H}), 6.90(\mathrm{~d}, J=8.7 \mathrm{~Hz}, 2 \mathrm{H}), 6.85(\mathrm{~d}, J=8.7 \mathrm{~Hz}, 2 \mathrm{H}) ;{ }^{13} \mathrm{C}$ NMR (75 MHz, $\left.\mathrm{CD}_{3} \mathrm{OD}\right): \delta$ 178.5, 165.2, 161.8, 136.3, 133.2, $130.3,117.1,116.6,111.5,95.7,87.2$. Anal. calcd for $\mathrm{C}_{15} \mathrm{H}_{10} \mathrm{O}_{3}$ : C, 75.62; H, 4.23. Found: C, 75.58; H, 4.20.

\section{Acknowledgements}

The authors are very grateful for economic support acquired from CONACyT (Project-255819) and PRODEP-SEP (ProjectDSA/103.5/16/10288). JAMS is grateful to the Royal Society (UK) for the International Alumni Grant.

\section{Notes and references}

1 (a) T. M. Shaikh, C.-M. Weng and F.-E. Hong, Coord. Chem. Rev., 2012, 256, 771; (b) V. K. Jain and L. Jain, Coord. Chem. Rev., 2005, 249, 3075.

2 T. Achard, Chimia, 2016, 70, 8.

3 (a) C.-Y. Hu, Y.-Q. Chen, G.-Y. Lin, M.-K. Huang, Y.-C. Chang and F.-E. Hong, Eur. J. Inorg. Chem., 2016, 3131; (b) Y.-C. Chang, C.-Y. Hu, Y.-H. Liang and F.-E. Hong, Polyhedron, 2016, 105, 123; (c) I. Cano, M. A. Huertos,
A. M. Chapman, G. Buntkowsky, T. Gutmann, P. B. Groszewicz and P. W. N. M. van Leeuwen, J. Am. Chem. Soc., 2015, 137, 7718; (d) E. Rafter, T. Gutmann, F. Löw, G. Buntkowsky, K. Philippot, B. Chaudret and P. W. N. M. van Leeuwen, Catal. Sci. Technol., 2013, 3, 595; (e) B. Kurscheid, B. Neumann, H.-G. Stammler and B. Hoge, Chem.-Eur. J., 2011, 17, 14935; (f) L. Ackermann, Synthesis, 2006, 1557.

4 L. Copey, L. Jean-Gérard, B. Andrioletti and E. Framery, Tetrahedron Lett., 2016, 57, 543.

5 (a) L. V. Graux, M. Giorgi, G. Buono and H. Clavier, Organometallics, 2015, 34, 1864; (b) N. P. N. Wellala and H. Guan, Org. Biomol. Chem., 2015, 13, 10802; (c) Y.-C. Chang, W.-C. Chang, C.-Y. Hu and F.-E. Hong, Organometallics, 2014, 33, 3523; (d) P. M. Castro, H. Gulyás, J. Benet-Buchholz, C. Bo, Z. Freixa and P. W. N. M. van Leeuwen, Catal. Sci. Technol., 2011, 1, 401; (e) D. Martin, D. Moraleda, T. Achard, L. Giordano and G. Buono, Chem.Eur. J., 2011, 17, 12729.

6 (a) J. Weismann, L. T. Scharf and V. H. Gessner, Organometallics, 2016, 35, 2507; (b) L. V. Graux, M. Giorgi, G. Buono and H. Clavier, Dalton Trans., 2016, 45, 6491; (c) D. Zell, S. Warratz, D. Gelman, S. J. Garden and L. Ackermann, Chem.-Eur. J., 2016, 22, 1248; (d) N. Allefeld, J. Bader, B. Neumann, H.-G. Stammler, N. Ignat'ev and B. Hoge, Inorg. Chem., 2015, 54, 7945; (e) A. Christiansen, D. Selent, A. Spannenberg, M. Köckerling, H. Reinke, W. Baumann, H. Jiao, R. Franke and A. Börner, Chem.-Eur. J., 2011, 17, 2120.

7 (a) J. Meeuwissen, R. J. Detz, A. J. Sandee, B. de Bruin and J. N. H. Reek, Dalton Trans., 2010, 39, 1929; (b) C. Waloch, J. Wieland, M. Keller and B. Breit, Angew. Chem., Int. Ed., 2007, 46, 3037; (c) B. Patel, S. J. A. Pope and G. Reid, Polyhedron, 1998, 17, 2345; (d) J. A. S. Duncan, T. A. Stephenson, M. D. Walkinshaw, D. Heden and D. M. Roundhill, J. Chem. Soc., Dalton Trans., 1984, 801; (e) J. A. S. Duncan, T. A. Stephenson, W. B. Beaulieu and D. M. Roundhill, J. Chem. Soc., Dalton Trans., 1983, 1755; (f) J. A. S. Duncan, D. Hedden, D. M. Roundhill, T. A. Stephenson and M. D. Walkinshaw, Angew. Chem., Int. Ed. Engl., 1982, 21, 452; (g) D. M. Roundhill, R. P. Sperline and W. B. Beaulieu, Coord. Chem. Rev., 1978, 26, 263.

8 (a) N. Allefeld, M. Grasse, N. Ignat'ev and B. Hoge, Chem.Eur. J., 2014, 20, 8615; (b) W.-J. Yoo and S. Kobayashi, Green Chem., 2013, 15, 1844; (c) B. Hoge, J. Bader, H. Beckers, Y. S. Kim, R. Eujen, H. Willner and N. Ignat'ev, Chem.-Eur. J., 2009, 15, 3567; (d) B. Hoge, P. Garcia, H. Willner and H. Oberhammer, Chem.-Eur. J., 2006, 12, 3567; (e) B. Hoge, S. Neufeind, S. Hettel, W. Wiebe and C. Thosen, J. Organomet. Chem., 2005, 690, 2382.

9 Y.-Y. Chang and F.-E. Hong, Tetrahedron, 2013, 69, 2327.

10 (a) H. Gulyás, I. Rivilla, S. Curreli, Z. Freixa and P. W. N. M. van Leeuwen, Catal. Sci. Technol., 2015, 5, 3822; (b) M. Karanik, D. Lesage, Y. Gimbert, P. Nava, S. Humbel, L. Giordano, G. Buono and J.-C. Tabet, Organometallics, 2011, 30, 4814; (c) T. J. Ahmed, B. R. Fox, 
S. M. M. Knapp, R. B. Yelle, J. J. Juliette and D. R. Tyler, Inorg. Chem., 2009, 48, 7828; (d) M. North, A. W. Parkins and A. N. Shariff, Tetrahedron Lett., 2004, 45, 7625; (e) X. Jiang, A. J. Minnaard, B. L. Feringa and J. G. de Vries, J. Org. Chem., 2004, 69, 2327; (f) L.-B. Hand, N. Choi and M. Tanaka, Organometallics, 1996, 15, 3259.

11 J. Coetzee, G. R. Eastham, A. M. Z. Slawin and D. J. ColeHamilton, Dalton Trans., 2014, 43, 3479.

12 H. Xu, K. Ekoue-Kovi and C. Wolf, J. Org. Chem., 2008, 73, 7638.

13 C. Wolf and R. Lerebours, Org. Lett., 2004, 6, 1147.

14 R. Lerebours and C. Wolf, Org. Lett., 2007, 9, 2737.

15 C. Wolf and K. Ekoue-Kovi, Eur. J. Org. Chem., 2006, 1917. 16 C. Wolf and R. Lerebours, J. Org. Chem., 2003, 68, 7077.

17 (a) J. A. Morales-Serna, A. Zúñiga-Martínez, M. Salmón, R. Gaviño and J. Cárdenas, Synthesis, 2012, 44, 446; (b) A. Sauza, J. A. Morales-Serna, M. García-Molina, R. Gaviño and J. Cárdenas, Synthesis, 2012, 44, 272.

18 K. R. Dixon and A. D. Rattray, Can. J. Chem., 1971, 49, 3997. 19 (a) P. Bergamini, V. Bertolasi, M. Cattabriga, V. Ferretti, U. Loprieno, N. Mantovani and L. Marvelli, Eur. J. Inorg. Chem., 2003, 5, 918; (b) T. G. Gebauer, K. Frenzen and Z. Dehnicke, Journal of Crystallography-Crystalline Materials, 1995, 210, 539; (c) T. Ghaffar, A. Kieszkiewicz, S. C. Nyburg and A. W. Parkins, Acta Crystallogr., Sect. C: Cryst. Struct. Commun., 1994, 50, 697.

20 B. M. Trost and J. T. Masters, Chem. Soc. Rev., 2016, 45, 2212. 21 For recent synthesis of 1,3-enynes, see: (a) N.-N. Wang, L.-R. Huang, W.-J. Hao, T.-S. Zhang, G. Li, S.-J. Tu and B. Jiang, Org. Lett., 2016, 18, 1298; (b) M. L. N. Rao, P. Dasgupta and V. N. Murty, RSC Adv., 2015, 5, 24834; (c) J.-Y. Jiao, X.-G. Zhang and X.-H. Zhang, Tetrahedron, 2015, 71, 9245; (d) A. Ikeda, M. Omote, K. Kusumoto, A. Tarui, K. Sato and A. Ando, Org. Biomol. Chem., 2015, 13, 8886; (e) J. H. Kim, Y. R. Jeong, S. L. Jeon and I. H. Jeong, J. Fluorine Chem., 2014, 167, 166; $(f)$ O. S. Morozov, A. F. Asachenko, D. V. Antonov, V. S. Kochurov, D. Y. Paraschuk and M. S. Nechaev, Adv. Synth. Catal., 2014, 356, 2671; $(g)$ P. Sun, H. Yan, L. Lu, D. Liu, G. Rong and J. Mao, Tetrahedron, 2013, 69, 6969; (h) Y. Zhu, T. Li, X. Qu, P. Sun, H. Yang and J. Mao, Org. Biomol. Chem., 2011, 9, 7309; (i) Y. Wen, A. Wang, H. Jiang, S. Zhu and L. Huang, Tetrahedron Lett., 2011, 52, 5736.

22 For reviews about Sonogashira reaction, see: $(a)$ L. Boulton, in Synthetic Methods in Drug Discovery, ed. D. C. Blakemore, P. M. Doyle and Y. M. Fobian, RSC, 2016, vol. 1, ch. 4, pp. 122-142; (b) M. B. Nielsen, Synthesis, 2016, 48, 2732; (c) M. Karak, L. C. A. Barbosa and G. C. Hargaden, RSC Adv., 2014, 4, 53442; (d) A. M. Thomas, A. Sujatha and G. Anilkumar, $R S C A d v$., 2014, 4, 21688; (e) D. Wang and S. Gao, Org. Chem. Front., 2014, 1, 556; $(f)$ M. Bakherad, Appl. Organomet. Chem., 2013, 27, 125; $(g)$ R. Chinchilla and C. Naájera, Chem. Soc. Rev., 2011, 40, 5084; (h) B. K. Singh, N. Kaval, S. Tomar, E. Van der Eycken and V. S. Parmar, Org. Process Res. Dev., 2008, 12, 468; (i) H. Doucet and J.-C. Hierso, Angew. Chem., Int. Ed., 2007, 46, 834 .
23 For some recent and selected examples about Sonogashira reaction, see: (a) H. Deol, S. Pramanik, M. Kumar, I. A. Khan and V. Bhalla, ACS Catal., 2016, 6, 3771; (b) V. N. Mikhaylov, V. N. Sorokoumov, K. A. Korvinson, A. S. Novikov and I. A. Balova, Organometallics, 2016, 35, 1684; (c) M. Gazvoda, M. Virant, A. Pevec, D. Urankar, A. Bolje, M. Kočevar and J. Košmrlj, Chem. Commun., 2016, 52, 157; (d) S. Pradhan, S. Dutta and R. P. John, New J. Chem., 2016, 40, 7140; (e) H. Salemi, B. Kaboudin, F. Kazemi and T. Yokomatsu, $R S C A d v$. , 2016, 6, 52656; $(f)$ C. I. Ezugwu, B. Mousavi, M. A. Asrafa, A. Mehta, H. Vardhan and F. Verpoort, Catal. Sci. Technol., 2016, 6, 2050; (g) A. M. Mak, Y. H. Lim, H. Jong, Y. Yang, C. W. Johannes, E. G. Robins and M. B. Sullivan, Organometallics, 2016, 35, 1036; (h) M. Trunk, A. Herrmann, H. Bildirir, A. Yassin, J. Schmidt and A. Thomas, Chem.-Eur. J., 2016, 22, 7179; (i) D.-C. Wang, H.-X. Wang, E.-J. Hao, X.-H. Jiang, M.-S. Xie, G.-R. Qu and H.-M. Guo, Adv. Synth. Catal., 2016, 358, 494; (j) M. Bagherzadeh, N. Mousavi, M. Zare, S. Jamali, A. Ellern and L. K. Woo, Inorg. Chim. Acta, 2016, 451, 227; (k) Y. Kelgokmen and M. n Zora, RSC Adv., 2016, 6, 4608.

24 For reviews about synthesis of ynones, see: (a) R. E. Whittaker, A. Dermenci and G. Dong, Synthesis, 2016, 48, 161; (b) C. F. Gers-Panther and T. J. J. Müller, Adv. Heterocycl. Chem., 2016, $120,67$.

25 For recent synthesis of ynones, see: (a) M. N. Soltani Rad, J. Saudi Chem. Soc., 2016, DOI: 10.1016/j.jscs.2016.06.001; (b) S. Tang, L. Zeng, Y. Liu and A. Lei, Angew. Chem., Int. Ed., 2015, 54, 15850; (c) H. Tan, H. Li, W. Ji and L. Wang, Angew. Chem., Int. Ed., 2015, 54, 8374; (d) X.-H. Ouyang, R.-J. Song, C.-Y. Wang, Y. Yang and J.-H. Li, Chem. Commun., 2015, 51, 14497; (e) R.-Y. Zhang, L.-Y. Xi, L. Zhang, S.-Y. Chen and X.-Q. Yu, Tetrahedron, 2015, 71, 6176; $(f)$ P.-F. Wang, Y.-S. Feng, Z.-F. Cheng, Q.-M. Wu, G.-Y. Wang, L.-L. Liu, J.-J. Dai, J. Xu and H.-J. Xu, J. Org. Chem., 2015, 80, 9314; (g) R.-Y. Zhang, L.-Y. Xi, L. Zhang, S.-Y. Chen and X.-Q. Yu, Tetrahedron, 2015, 71, 6176; $(h)$ C. L. Taylor and Y. Bolshan, J. Visualized Exp., 2015, 96, e52401, DOI: 10.3791/52401; (i) Z. Wang, L. Li and Y. Huang, J. Am. Chem. Soc., 2014, 136, 12233; (j) E. Mohammadi, B. Movassagh and M. Navidi, Helv. Chim. Acta, 2014, 97, 70; ( $k$ ) C. Taylor and Y. Bolshan, Org. Lett., 2014, 16, 488; ( l) J. A. Morales-Serna, A. Sauza, G. Padrón de Jesús, R. Gaviño, G. García de la Mora and J. Cárdenas, Tetrahedron Lett., 2013, 54, 7111.

26 R. J. Cox, D. J. Ritson, T. A. Dane, J. Berge, J. P. H. Charmantc and A. Kantacha, Chem. Commun., 2005, 1037.

27 U. J. Youn, Y. S. Lee, H. Jeong, J. Lee, J.-W. Nam, Y. J. Lee, E. S. Hwang, J.-H. Lee, D. Lee, S. S. Kang and E.-K. Seo, J. Nat. Prod., 2009, 72, 1895.

28 S. Karabulut, B. Özgün Öztürk and Y. Imamoglu, J. Organomet. Chem., 2010, 695, 2161.

29 O. V. Zatolochnaya, E. G. Gordeev, C. Jahier, V. P. Ananikov and V. Gevorgyan, Chem.-Eur. J., 2014, 20, 9578.

30 C. Xu, W. Du, Y. Zeng, B. Dai and H. Guo, Org. Lett., 2014, 16, 948. 
31 C. V. Ramana, P. Patel, K. Vanka, B. Miao and A. Degterev, Eur. J. Org. Chem., 2010, 5955.

32 X. Qu, T. Li, P. Sun, Y. Zhu, H. Yang and J. Mao, Org. Biomol. Chem., 2011, 9, 6938.

33 Y. Yamane, X. Liu, A. Hamasaki, T. Ishida, M. Haruta, T. Yokoyama and M. Tokunaga, Org. Lett., 2009, 11, 5162.

34 R. Bernini, S. Cacchi, G. Fabrizi, G. Forte, F. Petrucci, A. Prastaro, S. Niembro, A. Shafir and A. Vallribera, Green Chem., 2010, 12, 150.

35 F. Roschangar, J. Liu, E. Estanove, M. Dufour, S. Rodríguez, V. Farina, E. Hichey, A. Hossain, P.-J. Jones, H. Lee, B. Z. Lu, R. Varsolona, J. Schöder, P. Beaulieu, J. Gillard and C. H. Senayake, Tetrahedron Lett., 2008, 49, 363.
36 H.-J. Chen, Z.-Y. Lin, M.-Y. Li, R.-J. Lian, Q. W. Xue and J.-L. Chung, Tetrahedron, 2010, 66, 7755.

37 H. Kim and P. H. Lee, Adv. Synth. Catal., 2009, 351, 2827.

38 K. Park, G. Bae, J. Moon, J. Choe, K. Song and S. Lee, J. Org. Chem., 2010, 75, 6244.

39 B. Wang, M. Bonin and L. Micouin, J. Org. Chem., 2005, 70, 6126.

40 J.-Y. Chen, T.-C. Lin, S.-C. Chen, A.-J. Chen, C.-Y. Moub and F.-Y. Tsai, Tetrahedron, 2009, 65, 10134.

41 L. Zuo, S. Yao, W. Wang and W. Duan, Tetrahedron Lett., 2008, 49, 4054. 